\title{
Global Solutions of Stochastic Stackelberg Differential Games under Convex Control Constraint
}

\author{
Liangquan Zhang ${ }^{1 *}$, Wei Zhang ${ }^{1}$, \\ 1. School of Science \\ Beijing University of Posts and Telecommunications \\ Beijing 100876, China
}

August 12, 2021

\begin{abstract}
This paper is concerned with a Stackelberg stochastic differential game, where the systems are driven by stochastic differential equation (SDE for short), in which the control enters the randomly disturbed coefficients (drift and diffusion). The control region is postulated to be convex. By making use of the first-order adjoint equation (backward stochastic differential equation, BSDE for short), we are able to establish the Pontryagin's maximum principle for the leader's global Stackelberg solution, within adapted open-loop structure and closed-loop memoryless information one, respectively, where the term global indicates that the leader's domination over the entire game duration. Since the follower's adjoint equation turns out to be a BSDE, the leader will be confronted with a control problem where the state equation is a kind of fully coupled forward-backward stochastic differential equation (FBSDE for short).

As an application, we study a class of linear-quadratic (LQ for short) Stackelberg games in which the control process is constrained in a closed convex subset $\Gamma$ of full space $\mathbb{R}^{m}$. The state equations are represented by a class of fully coupled FBSDEs with projection operators on $\Gamma$. By means of monotonicity condition method, the existence and uniqueness of such FBSDEs are obtained. When the control domain is full space, we derive the resulting backward stochastic Riccati equations.
\end{abstract}

AMS subject classifications: 93E20, 60H15, 60H30.

Key words: Forward-backward stochastic differential equation, Linear-quadratic game, Monotonic condition, Maximum principle, Projection operator, Stackelberg differential game, Stochastic Riccati equation.

\section{Introduction}

H. von Stackelberg [1] first introduced a hierarchical solution for markets with leaders and followers in 1934 in order to obtain optimal strategies in competitive economics, which is now known as the Stackelberg equilibrium. The Stackelberg game is also know as the leader-follower game, whose economic background can be derived from some markets where certain companies have advantages of domination over others. Stackelberg strategies are rational and optimal solutions for both two players. A Stackelberg game can be described briefly as follows: the leader first announces his/her strategy at the beginning of the game. Based on the knowledge of the leader's action, the follower, makes an instantaneous reaction by optimizing his/her own

${ }^{*}$ L. Zhang acknowledges the financial support partly by the National Nature Science Foundation of China(Grant No. 11701040, 11871010 \&61871058) and the Fundamental Research Funds for the Central Universities (No. 2019XD-A11). E-mail: xiaoquan51011@163.com. 
performance index. Then, by anticipating the optimal response of the follower, the leader will select an optimal action to optimize his/her cost functional on the rational reaction curve of the follower. Therefore, one player must make a decision after the other player's decision is made. The leader's optimal action and the follower's rational response constitute a Stackelberg solution.

Since its nice structure and background in economy, there have been a great deal of substantial works along this research direction. For instance, the leader-follower's feature can be applied in many fields, such as the newsvendor/wholesaler problem (Øksendal et al. [2]), the optimal reinsurance problem (Chen and Shen [3]), the operations management and marketing channel problem (Li and Sethi [4) and the principal-agent/optimal contract problem (Cvitanić and Zhang [5]). Particularly, the feedback and adapted feedback information structures have been employed in treating supply chain management, marketing channel management, and economics problems in [6, 7, 8, 9, 10, 11] (for more information see reference therein). Besides, the celebrated Pontryagin's maximum principle for stochastic differential games within the framework of BSDE can be found in Wang and $\mathrm{Yu}$ [12, 13, and $\mathrm{Yu}$,14.

We state a few of them related our work. First, Castanon and Athans 15 considered an LQ stochastic dynamic Stackelberg strategies in the early, and obtained a feedback Stackelberg solution for two-person nonzero sum game. Yong [16] studied an indefinite LQ leader-follower stochastic differential game with random coefficients and control-dependent diffusion. The related Riccati equations for the follower and for the leader are derived sequentially to obtain the state feedback representation of the open-loop Stackelberg equilibrium points, moreover the sufficient conditions for their solvability with deterministic coefficients are given in the special case. Başar et al. [17] introduced the notion of mixed leadership in nonzero-sum differential games where one player could act as both leader and follower, depending on the control variable. Øksendal et al. 22 investigated a general stochastic Stackelberg differential game with delayed information, established the maximum principle, and applied it to continuous-time newsvendor problems. Bensoussan et al. [18] introduced several global solution concepts in terms of the players' information patterns, and derived the maximum principle by means of FBSDEs for the leader's global Stackelberg solution under the adapted open-loop and adapted closed-loop memoryless information structure (details see below) with non-controlled diffusion term. Meanwhile they investigate the LQ case where the weight matrices in the cost functionals are positive definite and controls do not entre into the diffusion term of the state equation. Mukaidani and $\mathrm{Xu}$ [19] considered the Stackelberg games for linear stochastic systems driven by Itô differential equations with multiple followers. The Stackelberg strategies, obtained by using sets of crosscoupled algebraic nonlinear matrix equations, are developed under two different settings: the followers act either cooperatively to attain Pareto optimality or non-cooperatively to arrive at a Nash equilibrium. Li and $\mathrm{Yu}$ 20 provided the solvability of a coupled FBSDEs under a multilevel self-similar domination-monotonicity structure, then it is employed to characterize the unique equilibrium of an LQ generalized Stackelberg stochastic differential game with hierarchy in a closed form. Huang et al. 21] studied a controlled linear-quadratic-Gaussian large population system combining major leader, minor leaders and minor followers. The Stackelberg-NashCournot (SNC for short) approximate equilibrium is obtained by means of the combination of a major-minor mean-field game and a leader-follower Stackelberg game, besides the feedback form of the SNC approximate equilibrium strategy is constructed through coupled Riccati equations.

In this paper, we shall study the Stackelberg games under two stochastic settings (taken from Benssousan et al. [18]). The first one is adapted open-loop (AOL) which can be states in summary as follows: given the strategy $u$ claimed by the leader at the beginning of the game, the follower wants to minimize his cost functional $J_{2}(u ; v)$ associated with the leader's strategy $u$ on the whole duration of the game. His optimal response $v^{*}$ will be an adapted process such that $J_{2}\left(u ; v^{*}(u)\right) \leq J_{2}(u ; v(u))$. The leader makes an instantaneous reaction $u^{*}$ by optimizing 
his/her performance index on the rational reaction curve of the follower, i.e., $J_{1}\left(u^{*} ; v^{*}\left(u^{*}\right)\right) \leq$ $J_{1}\left(u ; v^{*}(u)\right)$, anticipating the follower's optimal response $v^{*}$. The pair $\left(u^{*}, v^{*}\right)$ is called an AOL solution of the Stackelberg game. The other one, adapted closed-loop memoryless (ACLM), comparing with AOL, the leader's strategy and the follower's response strategy depend on the state (feedback form), which turns the control problem into a non-standard one. For leader's each strategy $u$ made in advanced, the follower would like to seek his optimal response $v^{*}$ such that $J_{2}\left(u ; v^{*}(u)\right) \leq J_{2}(u ; v(u))$. Then, by taking the rational response of the follower into account, the leader, of course, pick an action $u^{*}$ such that $J_{1}\left(u^{*} ; v^{*}\left(u^{*}\right)\right) \leq J_{1}\left(u ; v^{*}(u)\right)$. The pair $\left(u^{*}, v^{*}\right)$ is called an ACLM solution of the Stackelberg game.

To summarize the above, we see that the novelty of the formulation in this paper is the following:

- Comparing with Bensoussan et al. [18, our diffusion term in stochastic system allows to depend on control variable. As we shall see Section 2 below, due to this nice structure of our control system, on the one hand, the adjoint equation for leader becomes more general no matter of AOL or ACLM cases; on the other hand, the related stochastic Riccati equation for the follower and the leader considered simultaneously by putting the follower's Hamiltonian system as the leader's state equation will turn into a standard backward stochastic Riccati equation (see Tang [22]). Moreover, under certain assumptions, the stochastic Riccati equation admits a unique solution. For a general case, namely, nonconvex control set, see Section 4 .

- We focus on the LQ Stackelberg game with the control constrained in a closed convex set $\Gamma$ of full space: $\Gamma \subset \mathbb{R}^{m}$. One of the motivations to study the LQ problems with control constraint arises naturally from mathematical finance. For instance, the no-shorting constraint 11 in portfolio selection leads to the LQ control with positive control $\left(\Gamma=\mathbb{R}_{+}^{m}\right.$, the positive orthant). Moreover, since the general market accessibility constraint, it also promises interesting to investigate the LQ control with more general closed convex cone constraint (see [23]). As a response, this paper investigates the LQ Stackelberg game with general closed convex control constraint. The control constraint will bring some new features here: (1) The related Hamitonian system is no longer linear, and it becomes a class of nonlinear FBSDEs with projection operator. (2) Due to the nonlinearity, the standard Riccati equation with feedback control is no longer valid to represent the open-loop solution to the two-person leader-follower stochastic differential game.

The rest of the paper is organized as follows. Section 2 is devoted to presenting the maximum principle for a Stackelberg game of follower and leader under the AOL information pattern with convex control input, which is well known (cf. 24]). Based on previous result, we study Stackelberg games under the ACLM information pattern, and establish the maximum principle for the leader's optimal strategy, together with some other preliminary results. In section 3 , as applications, linear quadratic Stackelberg games under the AOL and ACLM information

\footnotetext{
${ }^{1}$ Short sales have so many risks that make it unsuitable for a novice investor. For starters, there is theoretically no limit to the investor's possible loss if the stock price rises instead of declines. A stock can only fall to zero, resulting in a $100 \%$ loss for a long investor, but there is no limit to how high a stock can theoretically go. A short seller who has not covered his or her position with a stop-loss buyback order can suffer tremendous losses if the stock price runs higher. For example, consider a company that becomes embroiled in scandal when its stock is trading at $\$ 70$ per share. An investor sees an opportunity to make a quick profit and sells the stock short at $\$ 65$. But then the company is able to quickly exonerate itself from the accusations by coming up with tangible proof to the contrary. The stock price quickly rises to $\$ 80$ a share, leaving the investor with a loss of $\$ 15$ per share for the moment. If the stock continues to rise, so do the investor's losses. Besides, short selling also involves significant expenses. There are the costs of borrowing the security to sell, the interest payable on the margin account that holds it, and trading commissions, etc.
} 
patterns are investigated, respectively. For former case, on the one hand, we prove the the existence and uniqueness of the solution to the associated Hamiltonian system for follower with projection operator; On the other hand, we show the existence and uniqueness of the solution to the associated stochastic Riccati equation under certain assumptions. For the latter case, we merely derive the associated Riccati equation which consists of a kind of complex FBSDEs, due to the quadratic and irregular feature. Some conclusions and unsolved issues for future research are displayed in Section 4. Some proof and discussion are displayed in Appendix.

\section{Preliminaries}

Throughout this paper, we denote by $\mathbb{R}^{n}$ the space of $n$-dimensional Euclidean space, by $\mathbb{R}^{n \times d}$ the space the matrices with order $n \times d$. Let $\left(\Omega, \mathcal{F},\left\{\mathcal{F}_{t}\right\}_{t \geq 0}, P\right)$ be a complete filtered probability space on which a 1-dimensional standard Brownian motion $W(\cdot)$ is defined, with $\left\{\mathcal{F}_{t}\right\}_{t \geq 0}$ being its natural filtration, augmented by all the $P$-null sets.

We now introduce the following spaces of processes:

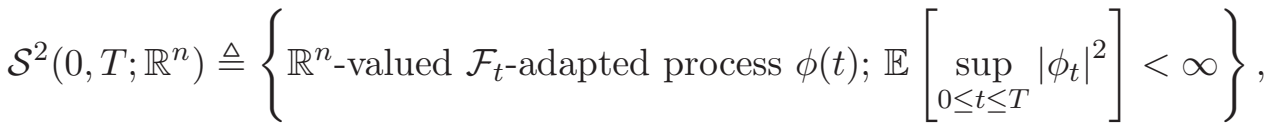

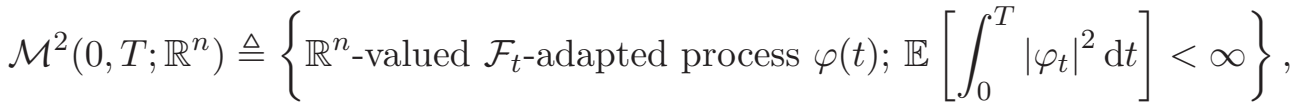

and denote $\mathcal{N}^{2}[0, T]=\mathcal{S}^{2}\left(0, T ; \mathbb{R}^{n}\right) \times \mathcal{S}^{2}\left(0, T ; \mathbb{R}^{n}\right) \times \mathcal{M}^{2}\left(0, T ; \mathbb{R}^{n}\right)$. Clearly, $\mathcal{N}^{2}[0, T]$ forms a Banach space.

Consider the following:

$$
\left\{\begin{aligned}
\mathrm{d} x(t) & =b(t, x(t), u(t), v(t)) \mathrm{d} t+\sigma(t, x(t), u(t), v(t)) \mathrm{d} W(t), \\
x(0) & =x_{0} \in \mathbb{R}^{n}
\end{aligned}\right.
$$

where and $(u(\cdot), v(\cdot))$ denotes the decisions of the leader and the follower, with values in subsets $U$ and $V$ in some closed convex subset $\Gamma_{1}$ and $\Gamma_{2}$ of full space $\mathbb{R}^{m_{1}}$ and $\mathbb{R}^{m_{2}}$, respectively.

The cost functionals for the leader and the follower to minimize are given, respectively, as follows:

$$
\mathcal{J}_{1}(u, v)=\mathbb{E}\left[\int_{0}^{T} l_{1}(t, x(t), u(t), v(t)) \mathrm{d} t+\Phi_{1}(x(T))\right]
$$

and

$$
\mathcal{J}_{2}(u, v)=\mathbb{E}\left[\int_{0}^{T} l_{2}(t, x(t), u(t), v(t)) \mathrm{d} t+\Phi_{2}(x(T))\right] .
$$

The coefficients $b$ and $\sigma$ in (11), and $l_{i}$ and $\Phi_{i}, i=1,2$ in the cost functionals (2) and (3) are specified as follows:

$$
\begin{aligned}
b & : \quad \Omega \times[0, T] \times \mathbb{R}^{n} \times \mathbb{R}^{m_{1}} \times \mathbb{R}^{m_{2}} \rightarrow \mathbb{R}^{n}, \mathcal{P} \times \mathcal{B}\left(\mathbb{R}^{n+m_{1}+m_{2}}\right) / \mathcal{B}\left(\mathbb{R}^{n}\right) \text { measurable, } \\
\sigma: & \Omega \times[0, T] \times \mathbb{R}^{n} \times \mathbb{R}^{m_{1}} \times \mathbb{R}^{m_{2}} \rightarrow \mathbb{R}^{n \times d}, \mathcal{P} \times \mathcal{B}\left(\mathbb{R}^{n+m_{1}+m_{2}}\right) / \mathcal{B}\left(\mathbb{R}^{n}\right) \text { measurable, } \\
l_{i}: & \Omega \times[0, T] \times \mathbb{R}^{n} \times \mathbb{R}^{m_{1}} \times \mathbb{R}^{m_{2}} \rightarrow \mathbb{R}, \mathcal{P} \times \mathcal{B}\left(\mathbb{R}^{n}\right) \times \mathcal{B}\left(\Gamma_{1}\right) \times \mathcal{B}\left(\Gamma_{2}\right) / \mathcal{B}(\mathbb{R}) \text { measurable, } \\
\Phi_{i}: & \Omega \times \mathbb{R}^{n} \rightarrow \mathbb{R}, \mathcal{F}_{T} \times \mathcal{B}\left(\mathbb{R}^{n}\right) / \mathcal{B}(\mathbb{R}) \text { measurable. }
\end{aligned}
$$

Letting $\varphi(t, x, u, v)=b(t, x, u, v), \sigma(t, x, u, v), l_{i}(t, x, u, v), \Phi_{i}, i=1,2$, we give the standing assumptions of our paper:

(A1) We postulate throughout the paper that $\varphi$ and its first and second derivatives are uniformly Lipschitz with respect to $(x, u, v)$ and $\varphi(\cdot, x, u, v) \in \mathcal{M}^{2}$, for $(x, u, v) \in \mathbb{R}^{n} \times \mathbb{R}^{m_{1}} \times$ $\mathbb{R}^{m_{2}}$. 
In this paper, we focus on the players' information structures $\eta$ as follows:

(a) adapted open-loop (AOL): $\eta(t)=\left\{x_{0}, \mathcal{F}_{t}\right\}, t \in[0, T]$,

(b) adapted closed-loop memoryless (ACLM): $\eta(t)=\left\{x_{0}, x(t), \mathcal{F}_{t}\right\}, t \in[0, T]$.

\section{$2.1 \quad$ AOL information structure}

For the AOL information structure, the admissible strategy spaces for the leader and the follower are denoted by

$$
\begin{gathered}
\mathcal{U}=\left\{u \mid u: \Omega \times[0, T] \rightarrow \Gamma_{1} \text { is } \mathcal{F}_{t^{-}} \text {-adapted satisfying } \mathbb{E}\left[\int_{0}^{T}|u(t)|^{2} \mathrm{~d} t\right]<\infty\right\}, \\
\mathcal{V}=\left\{v \mid v: \Omega \times[0, T] \times \mathcal{U} \rightarrow \Gamma_{2}, \text { for } u \in \mathcal{U}, v(\cdot, u) \text { is } \mathcal{F}_{t} \text {-adapted satisfying } \mathbb{E}\left[\int_{0}^{T}|v(t)|^{2} \mathrm{~d} t\right]<\infty\right\} .
\end{gathered}
$$

Remark 2.1 Since the initial state $x_{0}$ is commonly known by both players, $x_{0}$ is suppressed.

Under the AOL information pattern, we first establish a maximum principle for optimal control of the follower, whenever given the leader's strategy $u \in \mathcal{U}$.

Problem (AOL-F) Fix $u \in \mathcal{U}$. Seek an admissible control $v^{*}(\cdot) \in \mathcal{V}$ such that

$$
\mathcal{J}_{2}\left(u, v^{*}\right)=\inf _{v(\cdot) \in \mathcal{V}} \mathcal{J}_{2}(u, v)
$$

subject to (11).

Define the Hamiltonian function:

$$
\begin{aligned}
\mathcal{H}_{2}\left(t, x, u, v, p_{2}, q_{2}\right) & =\left\langle p_{2}, b(t, x, u, v)\right\rangle+\left\langle q_{2}, \sigma(t, x, u, v)\right\rangle+l_{2}(t, x, u, v), \\
\forall\left(t, x, u, v, p_{2}, q_{2}\right) & \in[0, T] \times \mathbb{R}^{n} \times \mathbb{R}^{m_{1}} \times \mathbb{R}^{m_{2}} \times \mathbb{R}^{n} \times \mathbb{R}^{n} .
\end{aligned}
$$

Then the maximum principle2 (cf. [25]) says that if we assume that $v^{*}(\cdot) \in \mathcal{V}$ is an optimal control, there exists a unique adapted solution $\left(p_{2}(\cdot), q_{2}(\cdot)\right) \in \mathcal{S}^{2}\left(0, T ; \mathbb{R}^{n}\right) \times \mathcal{M}^{2}\left(0, T ; \mathbb{R}^{n \times d}\right)$ such that

$$
\left\{\begin{aligned}
\mathrm{d} x(t) & =b\left(t, x(t), u(t), v^{*}(t)\right) \mathrm{d} t+\sigma\left(t, x(t), u(t), v^{*}(t)\right) \mathrm{d} W(t), \\
-\mathrm{d} p_{2}(t) & =\frac{\partial}{\partial x} \mathcal{H}_{2}\left(t, x(t), u(t), v^{*}(t), p_{2}(t), q_{2}(t)\right) \mathrm{d} t-q_{2}(t) \mathrm{d} W(t), \\
x(0) & =x_{0}, p_{2}(T)=\frac{\partial}{\partial x} \Phi_{2}(x(T)),
\end{aligned}\right.
$$

and

$$
v^{*}\left(t, x(t), u(t), p_{2}(t), q_{2}(t)\right)=\arg \min _{v \in \Gamma_{2}} \mathcal{H}_{2}\left(t, x(t), u(t), v, p_{2}(t), q_{2}(t)\right) .
$$

We suppose that, the function $v^{*}\left(t, x, u, p_{2}, q_{2}\right)$ is uniquely defined and is uniformly Lipschitz continuous with respect to $\left(x, u, p_{2}, q_{2}\right)$ and continuously differentiable 3 . Now inserting $v^{*}\left(t, x, u, p_{2}, q_{2}\right)$ into (4), we formulate the optimal control problem for leader.

Problem (AOL-L) Seek an admissible control $u^{*}(\cdot) \in \mathcal{U}$ such that

$$
\mathcal{J}_{1}\left(u^{*}, v^{*}\right)=\inf _{u(\cdot) \in \mathcal{U}} \mathcal{J}_{1}\left(u, v^{*}\right)
$$

\footnotetext{
${ }^{2}$ Since the control region is closed and convex, the first-order adjoint equation is needed. For general case, that is, compact control domain, the second-order adjoint equation must be introduced (see [25] and [26] for more details).

${ }^{3}$ In fact, we will see later in Section 3 in the framework of linear quadratic, whenever the control domain is closed and convex, by convex analysis, there indeed exists a unique optimal control in the form of projector.
} 
subject to

$$
\left\{\begin{aligned}
\mathrm{d} x(t)= & b\left(t, x(t), u(t), v^{*}\left(t, x(t), u(t), p_{2}(t), q_{2}(t)\right)\right) \mathrm{d} t \\
& +\sigma\left(t, x(t), u(t), v^{*}\left(t, x(t), u(t), p_{2}(t), q_{2}(t)\right)\right) \mathrm{d} W(t), \\
-\mathrm{d} p_{2}(t)= & \frac{\partial}{\partial x} \mathcal{H}_{2}\left(t, x(t), u(t), v^{*}\left(t, x(t), u(t), p_{2}(t), q_{2}(t)\right), p_{2}(t), q_{2}(t)\right) \mathrm{d} t \\
& -q_{2}(t) \mathrm{d} W(t), \\
x(0)= & x_{0}, p_{2}(T)=\frac{\partial}{\partial x} \Phi_{2}(x(T)) .
\end{aligned}\right.
$$

Remark 2.2 From Problem (AOL-L), reader maybe realize that one of the motivations to focus on fully coupled FBSDEs comes from stochastic Stackelberg differential games. In the literature, to treat fully coupled FBSDEs, Antonelli first provided a counterexample (see [27]) showing that the Lipschitz condition is not enough for the existence of FBSDEs in an arbitrarily large time duration. Thereby, more assumptions are essentially needed. To the best of our knowledge, there exist two approaches to deal with such FBSDEs. The first one is purely probabilistic (see [27, 28, 29]) under the monotone conditions; The second one concerns a kind of so called "foursteps scheme" method (combination of the methods of partial differential equation and probability or stochastic optimal control). Several major applications in mathematical finance have been made (see [30, 29]). It is now very clear that certain important problems in mathematical economics and mathematical finance, especially in the optimization problem, are formulated to be fully coupled FBSDEs (see [31]).

Clearly, for given $u(\cdot) \in \mathcal{U}$, FBSDEs (주) are fully coupled (while FBSDEs (3.2) in Bensoussan et al. [18 are partially coupled). In order to make the leader's problem well-posed, we proposed some conditions, mainly taken from Hu and Peng [28] (see also Peng and Wu 29]) to guarantee that FBSDEs (6) admit a unique adapted solution.

For $\Lambda^{1}=\left(x^{1}, p_{2}^{1}, q_{2}^{1}\right) \in \mathbb{R}^{n} \times \mathbb{R}^{n} \times \mathbb{R}^{n}, \Lambda^{2}=\left(x^{2}, p_{2}^{2}, q_{2}^{2}\right) \in \mathbb{R}^{n} \times \mathbb{R}^{n} \times \mathbb{R}^{n}$,

$$
\left[\Lambda^{1}, \Lambda^{2}\right]=\left\langle x^{1}, x^{2}\right\rangle+\left\langle p_{2}^{1}, p_{2}^{2}\right\rangle+\left\langle q_{2}^{1}, q_{2}^{2}\right\rangle,
$$

Let

$$
f_{2}\left(t, u, x, p_{2}, q_{2}\right)=\frac{\partial}{\partial x} \mathcal{H}_{2}\left(t, x, u, v^{*}\left(t, x, u, p_{2}, q_{2}\right), p_{2}, q_{2}\right)
$$

For $\Lambda=\left(x, p_{2}, q_{2}\right) \in \mathbb{R}^{n} \times \mathbb{R}^{n} \times \mathbb{R}^{n}$,

$$
F(t, u, \Lambda)=\left(f_{2}(t, u, \Lambda), b(t, u, \Lambda), \sigma(t, u, \Lambda)\right) .
$$

We assume

(A2) For each $\Lambda=\left(x, p_{2}, q_{2}\right) \in \mathbb{R}^{n} \times \mathbb{R}^{n} \times \mathbb{R}^{n}, F(t, u, \Lambda) \in \mathcal{M}^{2}\left(0, T ; \mathbb{R}^{n} \times \mathbb{R}^{n} \times \mathbb{R}^{n}\right)$, and for each $x \in \mathbb{R}^{n}, \frac{\partial}{\partial x} \Phi_{2}(x) \in L^{2}\left(\Omega, \mathcal{F}_{T} ; \mathbb{R}^{n}\right)$; there exists a constant $c_{1}>0$, such that

$$
\left|F\left(t, u, \Lambda^{1}\right)-F\left(t, u, \Lambda^{2}\right)\right| \leq c_{1}\left|\Lambda^{1}-\Lambda^{2}\right|, \Lambda^{i} \in \mathbb{R}^{n} \times \mathbb{R}^{n} \times \mathbb{R}^{n}, i=1,2
$$

and

$$
\left|\frac{\partial}{\partial x} \Phi_{2}\left(x_{1}\right)-\frac{\partial}{\partial x} \Phi_{2}\left(x_{2}\right)\right| \leq c_{1}\left|x_{1}-x_{2}\right|, P \text {-a.s. } x_{1}, x_{2} \in \mathbb{R}^{n} .
$$

(A3) There exists a constant $c_{2}>0$, such that

$$
\left[F\left(t, u, \Lambda^{1}\right)-F\left(t, u, \Lambda^{2}\right), \Lambda^{1}-\Lambda^{2}\right] \leq-c_{2}\left|\Lambda^{1}-\Lambda^{2}\right|^{2}, \Lambda^{i} \in \mathbb{R}^{n} \times \mathbb{R}^{n} \times \mathbb{R}^{n}, i=1,2
$$

and

$$
\left\langle\frac{\partial}{\partial x} \Phi_{2}\left(x_{1}\right)-\frac{\partial}{\partial x} \Phi_{2}\left(x_{2}\right), x_{1}-x_{2}\right\rangle \geq c_{2}\left|x_{1}-x_{2}\right|, P \text {-a.s. } x_{1}, x_{2} \in \mathbb{R}^{n} .
$$


Under (A2)-(A3), FBSDEs (6) have a unique adapted solution for $u \in \mathcal{U}$. Next, we will establish a maximum principle for leader's optimal control. Since the control domain is convex, the first adjoint equation is needed.

Proposition 2.1 Suppose that the Assumptions (A2)-(A3) hold. Let $u^{*}(\cdot)$ is an optimal strategy for the leader. Then there exists a unique adapted solution $\left(k(\cdot), p_{1}(\cdot), q_{1}(\cdot)\right) \in \mathcal{M}^{2}\left(0, T ; \mathbb{R}^{n} \times \mathbb{R}^{n} \times \mathbb{R}^{n}\right)$, such that

$$
u^{*}(t)=\arg \min _{u \in \mathcal{U}} \mathcal{H}_{1}\left(t, u, x(t), k(t), p_{1}(\cdot), p_{2}(\cdot), q_{1}(\cdot), q_{2}(\cdot)\right),
$$

where

$$
\begin{aligned}
\mathcal{H}_{1}\left(t, u, x, k, p_{1}, p_{2}, q_{1}, q_{2}\right)= & \left\langle p_{1}, b\left(t, x, u, v^{*}\left(t, x, u, p_{2}, q_{2}\right)\right)\right\rangle \\
& \left\langle q_{1}, \sigma\left(t, x, u, v^{*}\left(t, x, u, p_{2}, q_{2}\right)\right)\right\rangle \\
& +l_{1}\left(t, x, u, v^{*}\left(t, x, u, p_{2}, q_{2}\right)\right) \\
& -\left\langle k, f_{2}\left(t, u, x, p_{2}, q_{2}\right)\right\rangle
\end{aligned}
$$

and

$$
\left\{\begin{aligned}
\mathrm{d} k(t) & =-\frac{\partial}{\partial p_{2}} \mathcal{H}_{1} \mathrm{~d} t-\frac{\partial}{\partial q_{2}} \mathcal{H}_{1} \mathrm{~d} W(t), \\
\mathrm{d} p_{1}(t) & =\frac{\partial}{\partial x} \mathcal{H}_{1} \mathrm{~d} t+q_{1}(t) \mathrm{d} W(t), \\
k(0) & =0, p_{1}(T)=-\frac{\partial^{2}}{\partial x^{2}} \Phi_{2}(x(T)) k(T)+\frac{\partial}{\partial x} \Phi_{1}(x(T)),
\end{aligned}\right.
$$

where

$$
\begin{aligned}
& \frac{\partial}{\partial p_{2}} \mathcal{H}_{1}=\left(\frac{\partial b}{\partial v} \frac{\partial v^{*}}{\partial p_{2}}\right)^{\top} p_{1}+\left(\frac{\partial \sigma}{\partial v} \frac{\partial v^{*}}{\partial p_{2}}\right)^{\top} q_{1}+\left(\frac{\partial v^{*}}{\partial p_{2}}\right)^{\top} \frac{\partial l_{1}}{\partial v} \\
& -\frac{\partial b}{\partial x} k-\sum_{i=1}^{n} k_{i}\left(\frac{\partial v^{*}}{\partial p_{2}}\right)^{\top} \frac{\partial}{\partial v}\left(\frac{\partial b}{\partial x_{i}}\right)^{\top} p_{2} \\
& -\left(\frac{\partial^{2} l_{2}}{\partial v \partial x} \frac{\partial v^{*}}{\partial p_{2}}\right)^{\top} k-\sum_{i=1}^{n} k_{i}\left(\frac{\partial v^{*}}{\partial p_{2}}\right)^{\top} \frac{\partial}{\partial v}\left(\frac{\partial \sigma}{\partial x_{i}}\right)^{\top} q_{2}, \\
& \frac{\partial}{\partial q_{2}} \mathcal{H}_{1}=\left(\frac{\partial b}{\partial v} \frac{\partial v^{*}}{\partial q_{2}}\right)^{\top} p_{1}+\left(\frac{\partial \sigma}{\partial v} \frac{\partial v^{*}}{\partial q_{2}}\right)^{\top} q_{1}+\left(\frac{\partial v^{*}}{\partial q_{2}}\right)^{\top} \frac{\partial l_{1}}{\partial v} \\
& -\sum_{i=1}^{n} k_{i}\left(\frac{\partial v^{*}}{\partial q_{2}}\right)^{\top} \frac{\partial}{\partial v}\left(\frac{\partial b}{\partial x_{i}}\right)^{\top} p_{2} \\
& -\frac{\partial \sigma}{\partial x} k-\sum_{i=1}^{n} k_{i}\left(\frac{\partial v^{*}}{\partial q_{2}}\right)^{\top} \frac{\partial}{\partial v}\left(\frac{\partial \sigma}{\partial x_{i}}\right)^{\top} q_{2}-\left(\frac{\partial^{2} l_{2}}{\partial v \partial x} \frac{\partial v^{*}}{\partial q_{2}}\right)^{\top} k, \\
& \frac{\partial}{\partial x} \mathcal{H}_{1}=\left(\frac{\partial b}{\partial x}\right)^{\top} p_{1}+\left(\frac{\partial b}{\partial v} \frac{\partial v^{*}}{\partial x}\right)^{\top} p_{1}+\left(\frac{\partial \sigma}{\partial x}\right)^{\top} q_{1}+\left(\frac{\partial \sigma}{\partial v} \frac{\partial v^{*}}{\partial x}\right)^{\top} q_{1} \\
& +\frac{\partial l_{1}}{\partial x}+\left(\frac{\partial v^{*}}{\partial x}\right)^{\top} \frac{\partial l_{1}}{\partial v} \\
& -\sum_{i=1}^{n} k_{i}\left[\frac{\partial}{\partial x}\left(\frac{\partial b}{\partial x_{i}}\right)^{\top}+\left(\frac{\partial v^{*}}{\partial x_{2}}\right)^{\top} \frac{\partial}{\partial v}\left(\frac{\partial b}{\partial x_{i}}\right)^{\top}\right] p_{2} \\
& -\sum_{i=1}^{n} k_{i}\left[\frac{\partial}{\partial x}\left(\frac{\partial \sigma}{\partial x_{i}}\right)^{\top}+\left(\frac{\partial v^{*}}{\partial x_{2}}\right)^{\top} \frac{\partial}{\partial v}\left(\frac{\partial \sigma}{\partial x_{i}}\right)^{\top}\right] q_{2} \\
& -\left(\frac{\partial^{2} l_{2}}{\partial x^{2}}+\frac{\partial^{2} l_{2}}{\partial x \partial v} \frac{\partial v^{*}}{\partial x}\right)^{\top} k \text {. }
\end{aligned}
$$




\subsection{ACLM information structure}

For the ACLM information structure, the admissible strategy spaces for the leader and the follower are denoted by

$$
\begin{aligned}
\mathcal{U}= & \left\{u \mid u: \Omega \times[0, T] \times \mathbb{R}^{n} \rightarrow \Gamma_{1} \text { is } \mathcal{F}_{t^{-}} \text {-adapted for any } x \in \mathbb{R}^{n}, u(t, x)\right. \text { is continuously } \\
& \text { differentiable in } \left.x \text { for any }(t, x) \in \Omega \times[0, T] \text { satisfying }\left|\frac{\partial u}{\partial x}\right|<K\right\}, \\
\mathcal{V}= & \left\{v \mid: \Omega \times[0, T] \times \mathbb{R}^{n} \times \mathcal{U} \rightarrow \Gamma_{2} \text { is } \mathcal{F}_{t^{-}} \text {adapted for any } x \in \mathbb{R}^{n} \text { and } u \in \mathcal{U}\right\} .
\end{aligned}
$$

Now consider the following,

Problem (ACLM-F) For any $u \in \mathcal{U}$, seek an admissible control $v^{*}(\cdot) \in \mathcal{V}$ such that

$$
\mathcal{J}_{2}\left(u, v^{*}\right)=\inf _{v(\cdot) \in \mathcal{V}} \mathcal{J}_{2}(u, v)
$$

subject to

$$
\left\{\begin{aligned}
\mathrm{d} x(t) & =b(t, x(t), u(t, x(t)), v) \mathrm{d} t+\sigma(t, x(t), u(t, x(t)), v) \mathrm{d} W(t), \\
x(0) & =x_{0} .
\end{aligned}\right.
$$

Once again, then the maximum principle (cf. [25]) states that if we assume that $v^{*}(\cdot) \in \mathcal{V}$ is an optimal control, there exists a unique adapted solution $\left(p_{2}(\cdot), q_{2}(\cdot)\right) \in \mathcal{S}^{2}\left(0, T ; \mathbb{R}^{n}\right) \times$ $\mathcal{M}^{2}\left(0, T ; \mathbb{R}^{n \times d}\right)$ such that

$$
\left\{\begin{aligned}
-\mathrm{d} p_{2}(t) & =\frac{\partial}{\partial x} \mathcal{H}_{2}\left(t, x(t), u(t, x(t)), v(t), p_{2}(t), q_{2}(t)\right) \mathrm{d} t-q_{2}(t) \mathrm{d} W(t), \\
p_{2}(T) & =\frac{\partial}{\partial x} \Phi_{2}(x(T)),
\end{aligned}\right.
$$

with

$$
\begin{aligned}
& \frac{\partial}{\partial x} \mathcal{H}_{2}\left(t, x(t), u(t, x(t)), v(t), p_{2}(t), q_{2}(t)\right) \\
= & \left(\frac{\partial b}{\partial x}+\frac{\partial b}{\partial u} \frac{\partial u}{\partial x}\right)^{\top} p_{2}+\left(\frac{\partial \sigma}{\partial x}+\frac{\partial \sigma}{\partial u} \frac{\partial u}{\partial x}\right)^{\top} q_{2} \\
& +\frac{\partial l_{2}}{\partial x}+\left(\frac{\partial u}{\partial x}\right)^{\top} \frac{\partial l_{2}}{\partial u}
\end{aligned}
$$

and

$$
v^{*}\left(t, x(t), u, p_{2}(t), q_{2}(t)\right)=\arg \min _{v \in \Gamma_{2}} \mathcal{H}_{2}\left(t, x(t), u(t, x(t)), v, p_{2}(t), q_{2}(t)\right) .
$$

We now postulate that for any leader's strategy $u \in \mathcal{U}$, there exists a unique strategy $v^{*}$ for the follower that minimizes his cost functional $\mathcal{J}_{2}$ and that (10) yields $v^{*}(t)=v^{*}\left(t, x(t), u, p_{2}(t), q_{2}(t)\right)$. Then, taking into account the follower's optimal response, the leader will be taken action by solving the optimal control problem:

$$
\mathcal{J}_{1}\left(u^{*}, v^{*}\right)=\inf _{v(\cdot) \in \mathcal{V}} \mathcal{J}_{1}\left(u, v^{*}\right)
$$

subject to

$$
\left\{\begin{aligned}
\mathrm{d} x(t) & =b\left(t, x(t), u(t, x(t)), v^{*}(t)\right) \mathrm{d} t+\sigma\left(t, x(t), u(t, x(t)), v^{*}(t)\right) \mathrm{d} W(t), \\
-\mathrm{d} p_{2}(t) & =\frac{\partial}{\partial x} \mathcal{H}_{2}\left(t, x(t), u(t), v^{*}(t), p_{2}(t), q_{2}(t)\right) \mathrm{d} t-q_{2}(t) \mathrm{d} W(t), \\
x(0) & =x_{0}, p_{2}(T)=\frac{\partial}{\partial x} \Phi_{2}(x(T)),
\end{aligned}\right.
$$


with

$$
\begin{aligned}
& \frac{\partial}{\partial x} \mathcal{H}_{2}\left(t, x(t), u(t, x(t)), v^{*}(t), p_{2}(t), q_{2}(t)\right) \\
= & \left(\frac{\partial b}{\partial x}+\frac{\partial b}{\partial u} \frac{\partial u}{\partial x}\right)^{\top} p_{2}+\left(\frac{\partial \sigma}{\partial x}+\frac{\partial \sigma}{\partial u} \frac{\partial u}{\partial x}\right)^{\top} q_{2} \\
& +\frac{\partial l_{2}}{\partial x}+\left(\frac{\partial u}{\partial x}\right)^{\top} \frac{\partial l_{2}}{\partial u} .
\end{aligned}
$$

We assume that the follower has a unique optimal response strategy $v^{*}$ for every strategy $u \in \mathcal{U}$ of the leader. Similarly, we further suppose that the leader's problem is well-posed, i.e., for each $u \in \mathcal{U}$, there exists a unique triple $\left(x(\cdot), p_{2}(\cdot), q_{2}(\cdot)\right) \in \mathcal{N}^{2}[0, T]$ that solves FBSDEs (12). Clearly, the appearance of the derivative $\frac{\partial u}{\partial x}$ of the strategy $u$ in (12) leads to in a nonstandard optimal control problem for the leader. Employing the idea from Bensoussan et al. [18], we first transform the original issue to a standard stochastic optimal control problem, and subsequently establish the equivalence between the two in the sense that they coincide with the same optimal trajectory and cost.

Let us introduce the following optimal control problem:

$$
\begin{aligned}
\mathcal{J}\left(u_{1}^{*}, u_{2}^{*}\right) & =\min _{\left(u_{1}(\cdot), u_{2}(\cdot)\right)} \mathcal{J}_{1}(u, v) \\
& =\mathbb{E}\left[\int_{0}^{T} l_{1}\left(t, x(t), u_{2}(t) x(t)+u_{1}(t), \mu^{*}(t)\right) \mathrm{d} t+\Phi_{1}(x(T))\right],
\end{aligned}
$$

where

$$
\mu^{*}(t)=v^{*}\left(t, x(t), u_{2}(t) x(t)+u_{1}(t), p_{2}(t), q_{2}(t)\right)
$$

subject to

$$
\left\{\begin{aligned}
\mathrm{d} x(t)= & b\left(t, x(t), u_{2}(t) x(t)+u_{1}(t), \mu^{*}(t)\right) \mathrm{d} t \\
& +\sigma\left(t, x(t), u_{2}(t) x(t)+u_{1}(t), \mu^{*}(t)\right) \mathrm{d} W(t), \\
-\mathrm{d} p_{2}(t)= & \frac{\partial}{\partial x} \mathcal{H}_{2}\left(t, x(t), u_{2}(t) x(t)+u_{1}(t), \mu^{*}(t), p_{2}(t), q_{2}(t)\right) \mathrm{d} t-q_{2}(t) \mathrm{d} W(t), \\
x(0)= & x_{0}, p_{2}(T)=\frac{\partial}{\partial x} \Phi_{2}(x(T))
\end{aligned}\right.
$$

with

$$
\begin{aligned}
\frac{\partial}{\partial x} \mathcal{H}_{2}\left(t, x, u_{2} x+u_{1}, \mu^{*}, p_{2}, q_{2}\right)= & \left(\frac{\partial b}{\partial x}+\frac{\partial b}{\partial u} u_{2}\right)^{\top} p_{2}+\left(\frac{\partial \sigma}{\partial x}+\frac{\partial \sigma}{\partial u} u_{2}\right)^{\top} q_{2} \\
& -\frac{\partial l_{2}}{\partial x}-u_{2}^{\top} \frac{\partial l_{2}}{\partial u} .
\end{aligned}
$$

where $u_{2}$ and $u_{1}$ are adapted control processes with values in $\mathbb{R}^{m_{1}}$ and the ball $B_{K}\left(\mathbb{R}^{m_{1} \times n}\right)$ with radius $K$ in $\mathbb{R}^{m_{1}}$, respectively. We assume that the coefficients $\frac{\partial}{\partial x} \mathcal{H}_{2}\left(t, x, u_{2} x+u_{1}, \mu^{*}, p_{2}, q_{2}\right)$, $b, \sigma$ and $\frac{\partial}{\partial x} \Phi_{2}(x)$ satisfy the monotone condition (A1)-(A2). Thus, the problem (13)-(14) is well-posed.

Theorem 2.1 Assume that the above coefficients of the problem (13)-(14) satisfy the monotone conditions. Let $u^{*} \in \mathcal{U}$ be an optimal solution to the leader's problem (11)-(12) with the corresponding state trajectory $\left(\bar{x}(\cdot), \bar{p}_{2}(\cdot), \bar{q}_{2}(\cdot)\right) \in \mathcal{N}^{2}[0, T]$, then there exists a triple $\left(\chi(\cdot), p_{1}(\cdot), q_{1}(\cdot)\right) \in \mathcal{N}^{2}[0, T]$ such that

$$
\begin{aligned}
& \left(u^{*}(t, \bar{x}(t))-\frac{\partial u^{*}(t, \bar{x}(t))}{\partial x} \bar{x}(t), \frac{\partial u^{*}(t, \bar{x}(t))}{\partial x}\right) \\
= & \arg \min _{\left(u_{1}, u_{2}\right) \in \mathbb{R}^{m_{1} \times B_{K}\left(\mathbb{R}^{m_{1} \times n}\right)}} \mathcal{H}_{3}\left(t, u_{1}, u_{2}, \bar{x}(t), \chi(t), p_{1}(t), q_{1}(t), \bar{p}_{2}(t), \bar{q}_{2}(t)\right)
\end{aligned}
$$


subject to

$$
\left\{\begin{aligned}
\mathrm{d} \chi(t) & =-\frac{\partial}{\partial p_{2}} \mathcal{H}_{3} \mathrm{~d} t-\frac{\partial}{\partial q_{2}} \mathcal{H}_{3} \mathrm{~d} W(t) \\
\mathrm{d} p_{1}(t) & =\frac{\partial}{\partial x} \mathcal{H}_{3} \mathrm{~d} t+q_{1}(t) \mathrm{d} W(t) \\
\chi(0) & =0, p_{1}(T)=-\frac{\partial^{2} \Phi_{2}(\bar{x}(T))}{\partial x^{2}} \chi(T)+\frac{\partial \Phi_{1}(\bar{x}(T))}{\partial x}
\end{aligned}\right.
$$

where

$$
\begin{aligned}
& \mathcal{H}_{3}\left(t, u_{1}, u_{2}, \bar{x}, \chi, p_{1}, q_{1}, \bar{p}_{2}, \bar{q}_{2}\right) \\
= & \left\langle p_{1}, b\left(t, x, u_{2} x+u_{1}(t), v^{*}\left(t, x, u_{2} x+u_{1}, p_{2}, q_{2}\right)\right)\right\rangle \\
& +\left\langle q_{1}, \sigma\left(t, x, u_{2} x+u_{1}(t), v^{*}\left(t, x, u_{2} x+u_{1}, p_{2}, q_{2}\right)\right)\right\rangle \\
& -\left\langle\chi, \frac{\partial}{\partial x} \mathcal{H}_{2}\left(t, x, u_{2} x+u_{1}, \mu^{*}, p_{2}, q_{2}\right)\right\rangle \\
& +l_{1}\left(t, x, u_{2} x+u_{1}, v^{*}\left(t, x, u_{2} x+u_{1}, p_{2}, q_{2}\right)\right) .
\end{aligned}
$$

Here $\frac{\partial}{\partial p_{2}} \mathcal{H}_{3}, \frac{\partial}{\partial q_{2}} \mathcal{H}_{3}, \frac{\partial}{\partial x} \mathcal{H}_{3}$ are evaluated at the point

$$
\left(t, u^{*}(t, \bar{x}(t))-\frac{\partial u^{*}(t, \bar{x}(t))}{\partial x} \bar{x}(t), \frac{\partial u^{*}(t, \bar{x}(t))}{\partial x}, \bar{x}(t), \chi(t), p_{1}(t), q_{1}(t), \bar{p}_{2}(t), \bar{q}_{2}(t)\right) .
$$

For reader's convenience, we present a brief proof as follows:

Proof. Obviously, from the definitions of the optimal costs $\mathcal{J}$ and $\mathcal{J}_{1}$ associated with problems (11) and (13), we have $\mathcal{J}_{1} \leq \mathcal{J}$. Besides, based on $u^{*}$ of problem (11), we are able to structure a pair of control processes $\left(u_{1}^{*}, u_{2}^{*}\right)$ for problem (13) as follows:

$$
\begin{aligned}
u_{1}^{*}(t) & =u^{*}(t, \bar{x}(t))-\frac{\partial u^{*}(t, \bar{x}(t))}{\partial x} \bar{x}(t), \\
u_{2}^{*}(t) & =\frac{\partial u^{*}(t, \bar{x}(t))}{\partial x} .
\end{aligned}
$$

Employing these controls (16)-(17), the FBSDEs (14) admit the same solution as that of (12) with the optimal strategy $u^{*}$ from which we derive

$$
u^{*}(t, \bar{x}(t))=u_{2}^{*}(t) \bar{x}(t)+u_{1}^{*}(t) .
$$

Consequently, $\mathcal{J}=\mathcal{J}_{1}$ and the above constructed $\left(u_{1}^{*}, u_{2}^{*}\right)$ is an optimal control for problem (13), leading to the same state trajectory $\left(\chi(\cdot), p_{1}(\cdot), q_{1}(\cdot)\right)$. From the above arguments we state that

$$
\left(u_{1}^{*}(t)=u^{*}(t, \bar{x}(t))-\frac{\partial u^{*}(t, \bar{x}(t))}{\partial x} \bar{x}(t), \quad u_{2}^{*}(t)=\frac{\partial u^{*}(t, \bar{x}(t))}{\partial x}\right)
$$

is indeed an optimal control for problem (13), whenever $u^{*}$ is a solution for problem (11) with the corresponding forward state $\bar{x}(t)$. Therefore, we can establish the maximum principle for problem (11) of the leader via substituting (16)-(17) into the necessary conditions satisfied by the optimal control for problem (13).

Remark 2.3 Note that whenever $u$ doesn't contain the state $x$, we claim that Stackelberg solution is reduced to the AOL Stackelberg solution, and thus the maximum principle for both cases coincides. 


\section{Application to linear quadratic Stackelberg games}

In this section, the theoretical result obtained in Section 2 will be applied to study linear quadratic Stackelberg games under the AOL and ACLM information structures, respectively. Yong [16] derives stochastic Riccati equations for the follower and the leader sequentially with random coefficients and diffusion term of the state equation depending on controls, and the weight matrices in the cost functionals are not necessarily positive definite. To be precise, the follower gives his Riccati equation for any given strategy of the leader. Then the leader solves his problem involving a system of FBSDEs, whose coefficients depend on the solution of the follower's Riccati equation. Finally, a further analysis of the state feedback representation of the leader's optimal strategy provides the leader's Riccati equation. Under certain conditions, the solvability of the leader's Riccati equation in the case of deterministic coefficients is also discussed.

In contrast to Yong [16], we consider the similar system under convex control constraint . The systems will contain the projection operators which makes the system nonlinear (classical Riccati approach fails). When supposing the control set is full space, we may let the follower's Hamiltonian system as the leader's controlled state equation, and consequently, the state feedback representation of the AOL Stackelberg solution can be represented simultaneously for the leader and the follower. As a result, the corresponding Riccati equation is different from that in [16]. Moreover, by means of a linear transformation to a standard stochastic Riccati equation, we also prove that under certain conditions there exists a unique solution to the Riccati equation with stochastic coefficients studied by Tang 22. For a linear quadratic Stackelberg game under the ACLM case, we will see that the follower's Hamiltonian system is no longer linear, and that keeps us from deriving a Riccati equation if we handel the same way as in the AOL case. Instead, we postulate that the forward variable $\chi$ is linear with respect to the original state $x$, and then derive a kind of new but extremely complex FBSDEs which plays the same role as the Riccati equation in the AOL case.

\subsection{The AOL fashion}

To this end, let us introduce the state equation and the cost functional for leader and follower, respectively:

$$
\left\{\begin{aligned}
\mathrm{d} x(t)= & {\left[A(t) x(t)+B_{1}(t) u(t)+B_{2}(t) v(t)\right] \mathrm{d} t } \\
& +\left[C(t) x(t)+D_{1}(t) u(t)+D_{2}(t) v(t)\right] \mathrm{d} W(t) \\
x(0)= & x_{0} \in \mathbb{R}^{n}
\end{aligned}\right.
$$

The cost functionals for the leader and the follower to minimize are given, respectively, as follows:

$$
\mathcal{J}_{1}(u, v)=\frac{1}{2} \mathbb{E}\left[\int_{0}^{T}\left[\left(\left\langle Q_{1}(t) x(t), x(t)\right\rangle+\left\langle R_{1}(t) u(t), u(t)\right\rangle\right)\right] \mathrm{d} t+\left\langle\Phi_{1} x(T), x(T)\right\rangle\right]
$$

and

$$
\mathcal{J}_{2}(u, v)=\frac{1}{2} \mathbb{E}\left[\int_{0}^{T}\left(\left\langle Q_{2}(t) x(t), x(t)\right\rangle+\left\langle R_{2}(t) v(t), v(t)\right\rangle\right) \mathrm{d} t+\left\langle\Phi_{2} x(T), x(T)\right\rangle\right] .
$$

We make the following three assumptions on the coefficients of the above problem. 
(H1) Suppose that the matrix processes

$$
\begin{aligned}
A: & \Omega \times[0, T] \rightarrow \mathbb{R}^{n \times n}, \\
B_{1}: & \Omega \times[0, T] \rightarrow \mathbb{R}^{n \times m_{1}}, \\
B_{2}: & \Omega \times[0, T] \rightarrow \mathbb{R}^{n \times m_{2}}, \\
C & : \quad \Omega \times[0, T] \rightarrow \mathbb{R}^{n \times n}, \\
D_{1}: & \Omega \times[0, T] \rightarrow \mathbb{R}^{n \times m_{1}}, \\
D_{2}: & \Omega \times[0, T] \rightarrow \mathbb{R}^{n \times m_{2}}, \\
Q_{1}: & \Omega \times[0, T] \rightarrow \mathbb{R}^{n \times n}, \\
Q_{2} & : \Omega \times[0, T] \rightarrow \mathbb{R}^{n \times n}, \\
R_{1}: & \Omega \times[0, T] \rightarrow \mathbb{R}^{m_{1} \times m_{1}}, \\
R_{2} & : \quad \Omega \times[0, T] \rightarrow \mathbb{R}^{m_{2} \times m_{2}},
\end{aligned}
$$

and the random matrices $\Phi_{1}, \Phi_{2}: \Omega \rightarrow \mathbb{R}^{n}$ are uniformly bounded and $\left.\left\{\mathcal{F}_{t}, 0 \leq t \leq T\right\}\right\}$ adapted or $\mathcal{F}_{T}$-measurable.

(H2) Suppose that the state weighting matrix process $Q_{1}$ and $Q_{2}$ are a.s. a.e. symmetric and nonnegative. Also suppose that the terminal state weighting random matrix $\Phi_{1}$ and $\Phi_{2}$ are a.s. symmetric and nonnegative.

(H3) Suppose that the control weighting matrix process $R_{1}$ and $R_{2}$ are a.s. a.e. symmetric and uniformly positive.

The Hamiltonian function can be expressed by

$$
\begin{aligned}
\mathcal{H}_{2}\left(t, x, u, v, p_{2}, q_{2}\right)= & \left\langle p_{2}, A(t) x+B_{1}(t) u+B_{2}(t) v\right\rangle \\
& +\left\langle q_{2}, C(t) x+D_{1}(t) u+D_{2}(t) v\right\rangle \\
& +\frac{1}{2}\left[\left\langle Q_{2}(t) x, x\right\rangle+\left\langle R_{2}(t) v, v\right\rangle\right], \\
\forall\left(t, x, u, v, p_{2}, q_{2}\right) \in & {[0, T] \times \mathbb{R}^{n} \times \mathbb{R}^{m_{1}} \times \mathbb{R}^{m_{2}} \times \mathbb{R}^{n} \times \mathbb{R}^{n} . }
\end{aligned}
$$

The adjoint equation $\left(p_{2}(\cdot), q_{2}(\cdot)\right) \in \mathcal{S}^{2}\left(0, T ; \mathbb{R}^{n}\right) \times \mathcal{M}^{2}\left(0, T ; \mathbb{R}^{n \times d}\right)$ such that

$$
\left\{\begin{aligned}
-\mathrm{d} p_{2}(t) & =\left(A^{\top}(t) p_{2}(t)+C^{\top}(t) q_{2}(t)-Q_{2}(t) x(t)\right) \mathrm{d} t-q_{2}(t) W(t) \\
x(0) & =x_{0}, p_{2}(T)=-\Phi_{2} x(T)
\end{aligned}\right.
$$

Since $\Gamma_{2}$ is a closed convex set, then maximum principle reads as the following local form

$$
\left\langle-\frac{\partial \mathcal{H}_{2}}{\partial v}\left(t, x(t), u(t), v^{*}(t), p_{2}(t), q_{2}(t)\right), v-v^{*}(t)\right\rangle \leq 0, \quad \forall v \in \Gamma_{2} \text {, a.e. } t \in[0, T] \text {, P-a.s. }
$$

Hereafter, time argument is suppressed in case when no confusion occurs.

Noticing (21), then (23) yields that

$$
\left\langle-B_{2}^{\top} p_{2}-D_{2}^{\top} q_{2}-R_{2} v^{*}(t), v-v^{*}(t)\right\rangle \leq 0 \text {, for all } v \in \Gamma_{2} \text {, a.e. } t \in[0, T], P \text {-a.s. }
$$

or equivalently (noticing $R_{2}>0$ ),

$$
\left\langle R_{2}^{\frac{1}{2}}\left[-R_{2}^{-1}\left(B_{2}^{\top} p_{2}+D_{2}^{\top} q_{2}\right)-v^{*}(t)\right], R_{2}^{\frac{1}{2}}\left(v-v^{*}(t)\right)\right\rangle \leq 0, \forall v \in \Gamma_{2} \text {, a.e. } t \in[0, T], P \text {-a.s. }
$$


As $R_{2}(\cdot)>0$, we take the following norm on $\Gamma_{2} \subset \mathbb{R}^{m_{2}}$ (which is equivalent to its Euclidean norm)

$$
\|x\|_{R_{2}}^{2}=\langle\langle x, x\rangle\rangle:=\left\langle R_{2}^{\frac{1}{2}} x, R_{2}^{\frac{1}{2}} x\right\rangle,
$$

and by the well-known results of convex analysis, we obtain that (24) is equivalent to

$$
v^{*}(t)=\mathbf{P}_{\Gamma_{2}}\left[-R_{2}^{-1}(t)\left(B_{2}^{\top}(t) p_{2}(t)+D_{2}^{\top}(t) q_{2}(t)\right)\right], \quad \text { a.e. } t \in[0, T], P \text {-a.s. }
$$

where $\mathbf{P}_{\Gamma_{2}}(\cdot)$ is the projection mapping from $\mathbb{R}^{m_{2}}$ to its closed convex subset $\Gamma_{2}$ under the norm $\|\cdot\|_{R_{2}}$. For more details, see Appendix. From now on, we denote

$$
\varphi_{2}(t, p, q):=\mathbf{P}_{\Gamma_{2}}\left[-R_{2}^{-1}(t)\left(B_{2}^{\top}(t) p+D_{2}^{\top}(t) q\right)\right] .
$$

The follower's optimal strategy as follows:

$$
v^{*}(t)=\varphi_{2}\left(t, p_{1}(t), q_{1}(t)\right):=\mathbf{P}_{\Gamma_{2}}\left[-R_{2}^{-1}(t)\left(B_{2}^{\top}(t) p_{2}(t)+D_{2}^{\top}(t) q_{2}(t)\right)\right] .
$$

Now, we focus on the leader's problem. Her/His aim is to seek an optimal control $u^{*}(\cdot)$ such that

$$
\mathcal{J}_{1}\left(u^{*}, v^{*}\right)=\inf _{u(\cdot) \in \mathcal{U}} \mathcal{J}_{1}\left(u, v^{*}\right)
$$

subject to

$$
\left\{\begin{aligned}
\mathrm{d} x(t)= & {\left[A(t) x(t)+B_{1}(t) u(t)+B_{2}(t) \varphi_{2}\left(t, p_{2}(t), q_{2}(t)\right)\right] \mathrm{d} t } \\
& +\left[C(t) x(t)+D_{1}(t) u(t)+D_{2}(t) \varphi_{2}\left(t, p_{2}(t), q_{2}(t)\right)\right] \mathrm{d} W(t), \\
-\mathrm{d} p_{2}(t)= & {\left[A^{\top}(t) p_{2}(t)+C^{\top}(t) q_{2}(t)+Q_{2}(t) x(t)\right] \mathrm{d} t-q_{2}(t) W(t), } \\
x(0)= & x_{0}, p_{2}(T)=\Phi_{2} x(T) .
\end{aligned}\right.
$$

Obviously, FBSDEs (25) are fully coupled, which contains a nonlinear term $\varphi_{2}\left(t, p_{2}(t), q_{2}(t)\right)$. Nonetheless, under certain assumptions, we are able to prove the existence and uniqueness of such equations.

Theorem 3.1 Assume that (H1)-(H3) are in force. Then, for any given $u(\cdot) \in \mathcal{U}, F B S D E s$ (25) admit a unique adapted solution $\left(x(\cdot), p_{2}(\cdot), q_{2}(\cdot)\right) \in \mathcal{N}^{2}[0, T]$.

The proof can be found in the Appendix 4.

Remark 3.1 Due to the nonlinearity of (25), the classical approach of Riccati equation is not applicable in this case. Moreover, the methodology developed in Hu and Zhou [23] can not applied directly. On the one hand, the control domain there is postulated to be a closed cone involving the original point. From (5.2) in [23], we know that the optimal feedback control can be expressed as control process multiplying by the state variable. On the other hand, note that the liner system (18) is non-homogeneous linear equation, which doesn't satisfy the framework in [23] since the equations (5.24) and (5.25) in Hu and Zhou [23] can be represented explicitly. However, in our paper, we have two controls simultaneously, whose system, of course, is non-homogeneous.

Now we are ready to find the optimal control for leader. The leader's problem is well-posed since for every $u(\cdot) \in \mathcal{U}$, the FBSDEs (25) admits a unique solution. From Proposition 2.1, it is easy to derive the leader's optimal strategy as follows:

$$
u^{*}(t)=\varphi_{1}\left(t, p_{1}(t), q_{1}(t)\right):=\mathbf{P}_{\Gamma_{1}}\left[-R_{1}^{-1}(t)\left(B_{1}^{\top}(t) p_{1}(t)+D_{1}^{\top}(t) q_{1}(t)\right)\right],
$$


where

$$
\left\{\begin{aligned}
\mathrm{d} k(t) & =-\frac{\partial}{\partial p_{2}} \mathcal{H}_{1} \mathrm{~d} t-\frac{\partial}{\partial q_{2}} \mathcal{H}_{1} \mathrm{~d} W(t) \\
\mathrm{d} p_{1}(t) & =-\frac{\partial}{\partial x} \mathcal{H}_{1} \mathrm{~d} t+q_{1}(t) \mathrm{d} W(t), \\
k(0) & =0, p_{1}(T)=-\frac{\partial^{2}}{\partial x^{2}} \Phi_{2}(x(T)) k(T)+\frac{\partial}{\partial x} \Phi_{1}(x(T)),
\end{aligned}\right.
$$

and

$$
\begin{aligned}
& \mathcal{H}_{1}\left(t, u, x, k, p_{1}, p_{2}, q_{1}, q_{2}\right) \\
= & \left\langle p_{1}, A x+B_{1} u+B_{2} \varphi_{2}\left(t, p_{2}, q_{2}\right)\right\rangle \\
& +\left\langle q_{1}, C x+D_{1} u+D_{2} \varphi_{2}\left(t, p_{2}, q_{2}\right)\right\rangle \\
& +\frac{1}{2}\left(\left\langle Q_{1} x, x\right\rangle+\left\langle R_{1} u, u\right\rangle\right) \\
& -\left\langle k, A^{\top} p_{2}+C^{\top} q_{2}+Q_{2} x\right\rangle .
\end{aligned}
$$

From the uniqueness of the optimal strategy and Proposition A.1, we also know that the FBS-

DEs:

$$
\left\{\begin{aligned}
\mathrm{d} x(t)= & {\left[A(t) x(t)+B_{1}(t) u(t)+B_{2}(t) \varphi_{2}\left(t, p_{2}(t), q_{2}(t)\right)\right] \mathrm{d} t } \\
& +\left[C(t) x(t)+D_{1}(t) u(t)+D_{2}(t) \varphi_{2}\left(t, p_{2}(t), q_{2}(t)\right)\right] \mathrm{d} W(t), \\
-\mathrm{d} p_{2}(t)= & {\left[A^{\top}(t) p_{2}(t)+C^{\top}(t) q_{2}(t)+Q_{2}(t) x(t)\right] \mathrm{d} t-q_{2}(t) W(t), } \\
\mathrm{d} k(t)= & {\left[-B_{2}(t) \frac{\partial \varphi_{2}\left(t, p_{2}(t), q_{2}(t)\right)}{\partial p_{2}} p_{1}(t)-D_{2}(t) \frac{\partial \varphi_{2}\left(t, p_{2}(t), q_{2}(t)\right)}{\partial p_{2}} q_{1}(t)+A(t) k(t)\right] \mathrm{d} t } \\
& +\left[-B_{2}(t) \frac{\partial \varphi_{2}\left(t, p_{2}(t), q_{2}(t)\right)}{\partial q_{2}} p_{1}(t)-D_{2}(t) \frac{\partial \varphi_{2}\left(t, p_{2}(t), q_{2}(t)\right)}{\partial q_{2}} q_{1}(t)+C(t) k(t)\right] \mathrm{d} W(t), \\
-\mathrm{d} p_{1}(t)= & {\left[A^{\top}(t) p_{1}(t)+C^{\top}(t) q_{1}(t)+Q_{1}(t) x(t)-Q_{2}(t) k(t)\right] \mathrm{d} t-q_{1}(t) \mathrm{d} W(t), } \\
x(0)= & x_{0}, k(0)=0, p_{1}(T)=\Phi_{2} k(T)-\Phi_{1} x(T), p_{2}(T)=-\Phi_{2} x(T)
\end{aligned}\right.
$$

has a unique solution under the assumption $\frac{\partial}{\partial p_{2}} \varphi_{2}\left(t, p_{2}(t), q_{2}(t)\right)$ and $\frac{\partial}{\partial q_{2}} \varphi_{2}\left(t, p_{2}(t), q_{2}(t)\right)$ are well-defined.

Finally, we have the following coupled systems:

$$
\left\{\begin{aligned}
\mathrm{d} x(t)= & {\left[A(t) x(t)+B_{1}(t) \varphi_{1}\left(t, p_{1}(t), q_{1}(t)\right)+B_{2}(t) \varphi_{2}\left(t, p_{2}(t), q_{2}(t)\right)\right] \mathrm{d} t } \\
& +\left[C(t) x(t)+D_{1}(t) \varphi_{1}\left(t, p_{1}(t), q_{1}(t)\right)+D_{2}(t) \varphi_{2}\left(t, p_{2}(t), q_{2}(t)\right)\right] \mathrm{d} W(t), \\
-\mathrm{d} p_{2}(t)= & {\left[A^{\top}(t) p_{2}(t)+C^{\top}(t) q_{2}(t)+Q_{2}(t) x(t)\right] \mathrm{d} t-q_{2}(t) W(t), } \\
\mathrm{d} k(t)= & {\left[-B_{2}(t) \frac{\partial \varphi_{2}\left(t, p_{2}(t), q_{2}(t)\right)}{\partial p_{2}} p_{1}(t)-D_{2}(t) \frac{\partial \varphi_{2}\left(t, p_{2}(t), q_{2}(t)\right)}{\partial p_{2}} q_{1}(t)+A(t) k(t)\right] \mathrm{d} t } \\
& +\left[-B_{2}(t) \frac{\partial \varphi_{2}\left(t, p_{2}(t), q_{2}(t)\right)}{\partial q_{2}} p_{1}(t)-D_{2}(t) \frac{\partial \varphi_{2}\left(t, p_{2}(t), q_{2}(t)\right)}{\partial q_{2}} q_{1}(t)+C(t) k(t)\right] \mathrm{d} W(t), \\
-\mathrm{d} p_{1}(t)= & {\left[A^{\top}(t) p_{1}(t)+C^{\top}(t) q_{1}(t)+Q_{1}(t) x(t)-Q_{2}(t) k(t)\right] \mathrm{d} t-q_{1}(t) \mathrm{d} W(t), } \\
x(0)= & x_{0}, k(0)=0, p_{1}(T)=\Phi_{2} k(T)-\Phi_{1} x(T), p_{2}(T)=-\Phi_{2} x(T)
\end{aligned}\right.
$$

Next we set $\Gamma_{1}=\mathbb{R}^{m_{1}}, \Gamma_{2}=\mathbb{R}^{m_{2}}$. We observe that the Riccati equation approach is really applicable in this case, and the AOL Stackelberg solution $\left(u^{*}, v^{*}\right)$ can be written as

$$
\left\{\begin{array}{l}
u^{*}(t)=-R_{1}^{-1}(t)\left(B_{1}^{\top}(t) p_{1}(t)+D_{1}^{\top}(t) q_{1}(t)\right), \\
v^{*}(t)=-R_{2}^{-1}(t)\left(B_{2}^{\top}(t) p_{2}(t)+D_{2}^{\top}(t) q_{2}(t)\right) .
\end{array}\right.
$$

In this case, FBSDEs (28) turn into

$$
\left\{\begin{aligned}
\mathrm{d} x(t)= & {\left[A(t) x(t)+B_{1}(t) \varphi_{1}\left(t, p_{1}(t), q_{1}(t)\right)+B_{2}(t) \varphi_{2}\left(t, p_{2}(t), q_{2}(t)\right)\right] \mathrm{d} t } \\
& +\left[C(t) x(t)+D_{1}(t) \varphi_{1}\left(t, p_{1}(t), q_{1}(t)\right)+D_{2}(t) \varphi_{2}\left(t, p_{2}(t), q_{2}(t)\right)\right] \mathrm{d} W(t), \\
\mathrm{d} k(t)= & {\left[B_{2}(t) R_{2}^{-1}(t) B_{2}^{\top}(t) p_{1}(t)+D_{2}(t) R_{2}^{-1}(t) B_{2}^{\top}(t) q_{1}(t)+A(t) k(t)\right] \mathrm{d} t } \\
& +\left[B_{2}(t) R_{2}^{-1}(t) D_{2}^{\top}(t) p_{1}(t)+D_{2}(t) R_{2}^{-1}(t) D_{2}^{\top}(t) q_{1}(t)+C(t) k(t)\right] \mathrm{d} W(t), \\
-\mathrm{d} p_{1}(t)= & {\left[A^{\top}(t) p_{1}(t)+C^{\top}(t) q_{1}(t)+Q_{1}(t) x(t)-Q_{2}(t) k(t)\right] \mathrm{d} t-q_{1}(t) \mathrm{d} W(t), } \\
-\mathrm{d} p_{2}(t)= & {\left[A^{\top}(t) p_{2}(t)+C^{\top}(t) q_{2}(t)+Q_{2}(t) x(t)\right] \mathrm{d} t-q_{2}(t) W(t), } \\
x(0)= & x_{0}, k(0)=0, p_{1}(T)=-\Phi_{2} k(T)+\Phi_{1} x(T), p_{2}(T)=\Phi_{2} x(T) .
\end{aligned}\right.
$$


It is possible to derive the feedback representation of the Stackelberg solution $\left(u^{*}, v^{*}\right)$ in terms of the state $(x, k)$. To this end, we introduce the following notations for simplicity,

$$
X=\left(\begin{array}{c}
x \\
k
\end{array}\right), P=\left(\begin{array}{l}
p_{1} \\
p_{2}
\end{array}\right), Q=\left(\begin{array}{l}
q_{1} \\
q_{2}
\end{array}\right)
$$

and

$$
\begin{aligned}
\mathcal{A} & =\left(\begin{array}{cc}
A & 0 \\
0 & A
\end{array}\right), \mathcal{C}=\left(\begin{array}{cc}
C & 0 \\
0 & C
\end{array}\right), X_{0}=\left(\begin{array}{c}
x_{0} \\
0
\end{array}\right), \\
\mathcal{B}_{1} & =\left(\begin{array}{cc}
B_{1} R_{1}^{-1} B_{1}^{\top} & B_{2} R_{2}^{-1} B_{2}^{\top} \\
-B_{2} R_{2}^{-1} B_{2}^{\top} & 0
\end{array}\right), \mathcal{B}_{2}=\left(\begin{array}{cc}
B_{1} R_{1}^{-1} D_{1}^{\top} & B_{2} R_{2}^{-1} D_{2}^{\top} \\
-D_{2} R_{2}^{-1} B_{2}^{\top} & 0
\end{array}\right), \\
\mathcal{D}_{1} & =\left(\begin{array}{ccc}
D_{1} R_{1}^{-1} B_{1}^{\top} & D_{2} R_{2}^{-1} B_{2}^{\top} \\
-B_{2} R_{2}^{-1} D_{2}^{\top} & 0
\end{array}\right), \mathcal{D}_{2}=\left(\begin{array}{cc}
D_{1} R_{1}^{-1} D_{1}^{\top} & D_{2} R_{2}^{-1} D_{2}^{\top} \\
-D_{2} R_{2}^{-1} D_{2}^{\top} & 0
\end{array}\right), \\
Q_{1} & =\left(\begin{array}{cc}
Q_{1} & -Q_{2} \\
Q_{2} & 0
\end{array}\right), \Phi_{1}=\left(\begin{array}{cc}
\Phi_{1} & -\Phi_{2} \\
\Phi_{2} & 0
\end{array}\right) .
\end{aligned}
$$

Then, FBSDEs (28) can be rewritten as

$$
\left\{\begin{aligned}
\mathrm{d} X(t)= & {\left[\mathcal{A}(t) X(t)-\mathcal{B}_{1}(t) P(t)-\mathcal{B}_{2}(t) Q(t)\right] \mathrm{d} t } \\
& +\left[\mathcal{C}(t) X(t)-\mathcal{D}_{1}(t) P(t)-\mathcal{D}_{2}(t) Q(t)\right] \mathrm{d} W(t), \\
-\mathrm{d} P(t)= & {\left[\mathcal{A}(t) P(t)+\mathcal{C}^{\top}(t) Q(t)+Q_{1}(t) X(t)\right] \mathrm{d} t-Q(t) \mathrm{d} W(t), } \\
X(0)= & X_{0}, P(T)=\hat{\Phi} X(T) .
\end{aligned}\right.
$$

We are ready to derive the Riccati equation. To this end, assume that there exists a matrixvalued process $\mathcal{P}$ such that

$$
P(t)=\mathcal{R}(t) X(t), t \in[0, T],
$$

where $\mathcal{R}(t)$ is an $\mathcal{F}_{t}$-adapted process with values in $\mathbb{R}^{n \times n}$. In general, $\mathcal{R}(t)$ is not a bounded variation function with respect to $t$. We tentatively assume that $\mathcal{R}(t)$ is a semi-martingale

$$
\mathcal{R}(t)=\Phi_{1}+\int_{t}^{T} \Pi(s) \mathrm{d} s-\int_{t}^{T} \Psi(s) \mathrm{d} W(s), 0 \leq t \leq T,
$$

Applying the Itô's formula to $\mathcal{R}(\cdot) X(\cdot)$, we obtain

$$
\begin{aligned}
& \mathcal{R}(t)\left[\mathcal{A}(t) X(t)-\mathcal{B}_{1}(t) \mathcal{R}(t) X(t)-\mathcal{B}_{2}(t) Q(t)\right] \mathrm{d} t \\
& -\Pi(t) X(t) \mathrm{d} t+\Psi(t)\left[\mathcal{C}(t) X(t)-\mathcal{D}_{1}(t) \mathcal{R}(t) X(t)-\mathcal{D}_{2}(t) Q(t)\right] \mathrm{d} t \\
& +\mathcal{R}(t)\left[\mathcal{C}(t) X(t)-\mathcal{D}_{1}(t) \mathcal{R}(t) X(t)-\mathcal{D}_{2}(t) Q(t)\right] \mathrm{d} W(t)+\Psi(t) X(t) \mathrm{d} W(t) \\
= & \mathrm{d} P(t) \\
= & -\left[\mathcal{A}(t) \mathcal{R}(t) X(t)+\mathcal{C}^{\top}(t) Q(t)+Q_{1}(t) X(t)\right] \mathrm{d} t+Q(t) \mathrm{d} W(t) .
\end{aligned}
$$

It is easy to see

$$
Q(t)=\mathcal{R}(t)\left[\mathcal{C}(t) X(t)-\mathcal{D}_{1}(t) \mathcal{R}(t) X(t)-\mathcal{D}_{2}(t) Q(t)\right]+\Psi(t) X(t),
$$

from which we get

$$
Q(t)=\Xi(t) X(t)
$$

where

$$
\Xi(t)=\left(I+\mathcal{R}(t) \mathcal{D}_{2}(t)\right)^{-1}\left[\mathcal{R}(t) \mathcal{C}(t)-\mathcal{R}(t) \mathcal{D}_{1}(t) \mathcal{R}(t)+\Psi(t)\right]
$$


Inserting (33) into (32), we have

$$
\begin{aligned}
& \mathcal{R}(t)\left[\mathcal{A}(t)-\mathcal{B}_{1}(t) \mathcal{R}(t)-\mathcal{B}_{2}(t) \Xi(t)\right] X(t) \mathrm{d} t \\
& -\Pi(t) X(t) \mathrm{d} t+\Psi(t)\left[\mathcal{C}(t)-\mathcal{D}_{1}(t) \mathcal{R}(t)-\mathcal{D}_{2}(t) \Xi(t)\right] X(t) \mathrm{d} t \\
= & -\left[\mathcal{A}(t) \mathcal{R}(t)+\mathcal{C}^{\top}(t) \Xi(t)+Q_{1}(t)\right] X(t) \mathrm{d} t,
\end{aligned}
$$

which yields

$$
\begin{aligned}
\Pi(t)= & \mathcal{A}(t) \mathcal{R}(t)+\mathcal{C}^{\top}(t) \Xi(t)+Q_{1}(t) \\
& +\mathcal{R}(t)\left[\mathcal{A}(t)-\mathcal{B}_{1}(t) \mathcal{R}(t)-\mathcal{B}_{2}(t) \Xi(t)\right] \\
& +\Psi(t)\left[\mathcal{C}(t)-\mathcal{D}_{1}(t) \mathcal{R}(t)-\mathcal{D}_{2}(t) \Xi(t)\right]
\end{aligned}
$$

Consequently, we obtain

$$
\begin{aligned}
\mathrm{d} \mathcal{R}(t)= & {\left[\mathcal{A}(t) \mathcal{R}(t)+\mathcal{C}^{\top}(t) \Xi(t)+Q_{1}(t)\right.} \\
& +\mathcal{R}(t)\left[\mathcal{A}(t)-\mathcal{B}_{1}(t) \mathcal{R}(t)-\mathcal{B}_{2}(t) \Xi(t)\right] \\
& \left.+\Psi(t)\left[\mathcal{C}(t)-\mathcal{D}_{1}(t) \mathcal{R}(t)-\mathcal{D}_{2}(t) \Xi(t)\right]\right] \mathrm{d} t \\
& -\Psi(t) \mathrm{d} W(t) \\
\mathcal{R}(T)= & \Phi_{1} .
\end{aligned}
$$

Remark 3.2 Whenever $D_{1}(t)=D_{2}(t)=0$, the Riccati equation (34) becomes the right form (5.21) in Bensoussan et al. [18].

We should claim that the Riccati equation (34) is just another equivalent form in Tang 22] (see Discussion in Appendix). Nonetheless, the coefficients $\mathcal{B}_{1}, \mathcal{B}_{2}, \mathcal{D}_{1}, \mathcal{D}_{2}$ and $Q_{1}$ are not symmetric matrices. Next, we shall introduce a linear transformation to turn (34) into a standard Riccati equation for $n=1$.

Theorem 3.2 For $n=1$, we assume that

$$
\begin{aligned}
\frac{Q_{2}}{Q_{1}} & =\frac{\Phi_{2}}{\Phi_{1}}, \\
\frac{B_{2} R_{2}^{-1} B_{2}^{\top}}{B_{1} R_{1}^{-1} B_{1}^{\top}} & =\frac{B_{2} R_{2}^{-1} D_{2}^{\top}}{B_{1} R_{1}^{-1} D_{1}^{\top}}=\frac{D_{2} R_{2}^{-1} B_{2}^{\top}}{D_{1} R_{1}^{-1} B_{1}^{\top}}=\frac{D_{2} R_{2}^{-1} D_{2}^{\top}}{D_{1} R_{1}^{-1} D_{1}^{\top}}
\end{aligned}
$$

are in force. Then the Riccati equation (34) admits a unique solution.

Proof. Let

$$
\begin{aligned}
\frac{Q_{2}}{Q_{1}} & =\frac{\Phi_{2}}{\Phi_{1}}=\lambda, \\
\frac{B_{2} R_{2}^{-1} B_{2}^{\top}}{B_{1} R_{1}^{-1} B_{1}^{\top}} & =\frac{B_{2} R_{2}^{-1} D_{2}^{\top}}{B_{1} R_{1}^{-1} D_{1}^{\top}}=\frac{D_{2} R_{2}^{-1} B_{2}^{\top}}{D_{1} R_{1}^{-1} B_{1}^{\top}}=\frac{D_{2} R_{2}^{-1} D_{2}^{\top}}{D_{1} R_{1}^{-1} D_{1}^{\top}}=\mu .
\end{aligned}
$$

Let us introduce a linear transformation

$$
X=\bar{X}, P=\Upsilon \bar{P}, Q=\Upsilon \bar{Q},
$$

via a matrix $\Upsilon$ (determined later). So The FBSDEs (30) can be expressed as

$$
\left\{\begin{aligned}
\mathrm{d} \bar{X}(t)= & {\left[\overline{\mathcal{A}}(t) \bar{X}(t)-\overline{\mathcal{B}}_{1}(t) \bar{P}(t)-\overline{\mathcal{B}}_{2}(t) \bar{Q}(t)\right] \mathrm{d} t } \\
& +\left[\overline{\mathcal{C}}(t) \bar{X}(t)-\overline{\mathcal{D}}_{1}(t) \bar{P}(t)-\overline{\mathcal{D}}_{2}(t) \bar{Q}(t)\right] \mathrm{d} W(t), \\
-\mathrm{d} \bar{P}(t)= & {\left[\overline{\mathcal{A}}(t) \bar{P}(t)+\overline{\mathcal{C}}^{\top}(t) \bar{Q}(t)+\bar{Q}_{1}(t) \bar{X}(t)\right] \mathrm{d} t-Q(t) \mathrm{d} W(t), } \\
\bar{X}(0)= & X_{0}, \bar{P}(T)=\bar{\Phi}_{1} \bar{X}(T),
\end{aligned}\right.
$$


where

$$
\begin{aligned}
\overline{\mathcal{A}} & =\Upsilon^{-1} A \Upsilon, \overline{\mathcal{B}}_{1}=\mathcal{B}_{1} \Upsilon, \overline{\mathcal{B}}_{2}=\mathcal{B}_{2} \Upsilon \\
\overline{\mathcal{C}} & =\Upsilon^{-1} C \Upsilon, \overline{\mathcal{D}}_{1}=\mathcal{D}_{1} \Upsilon, \overline{\mathcal{D}}_{2}=\mathcal{D}_{2} \Upsilon \\
\bar{Q}_{1} & =\Upsilon^{-1} Q_{1}, \bar{\Phi}_{1}=\Upsilon^{-1} \Phi_{1} .
\end{aligned}
$$

Note that $A, C$ are symmetric metrics. We are going to seek $\Upsilon$ such that $\overline{\mathcal{B}}_{1}, \overline{\mathcal{B}}_{2}, \overline{\mathcal{D}}_{1}, \overline{\mathcal{D}}_{2}, \bar{Q}_{1}$ and $\bar{\Phi}_{1}$ are symmetric. From the assumption (35), it is easy to compute that

$$
\Upsilon=\left(\begin{array}{cc}
1 & -2 \mu \\
2 \lambda & 1
\end{array}\right)
$$

Therefore, $\overline{\mathcal{A}}=\mathcal{A}, \overline{\mathcal{C}}=\mathcal{C}$,

$$
\begin{aligned}
& \overline{\mathcal{B}}_{1}=\left(\begin{array}{cc}
B_{1} R_{1}^{-1} B_{1}^{\top}+2 \lambda B_{2} R_{2}^{-1} B_{2}^{\top} & -B_{2} R_{2}^{-1} B_{2}^{\top} \\
-B_{2} R_{2}^{-1} B_{2}^{\top} & 2 \mu B_{2} R_{2}^{-1} B_{2}^{\top}
\end{array}\right), \\
& \overline{\mathcal{B}}_{2}=\left(\begin{array}{cc}
B_{1} R_{1}^{-1} D_{1}^{\top}+2 \lambda B_{2} R_{2}^{-1} D_{2}^{\top} & -B_{2} R_{2}^{-1} B_{2}^{\top} \\
-D_{2} R_{2}^{-1} B_{2}^{\top} & 2 \mu D_{2} R_{2}^{-1} B_{2}^{\top}
\end{array}\right), \\
& \overline{\mathcal{D}}_{1}=\left(\begin{array}{cc}
D_{1} R_{1}^{-1} B_{1}^{\top}+2 \lambda D_{2} R_{2}^{-1} B_{2}^{\top} & -B_{2} R_{2}^{-1} D_{2}^{\top} \\
-B_{2} R_{2}^{-1} D_{2}^{\top} & 2 \mu B_{2} R_{2}^{-1} D_{2}^{\top}
\end{array}\right), \\
& \overline{\mathcal{D}}_{2}=\left(\begin{array}{cc}
D_{1} R_{1}^{-1} D_{1}^{\top}+2 \lambda D_{2} R_{2}^{-1} D_{2}^{\top} & -D_{2} R_{2}^{-1} D_{2}^{\top} \\
-D_{2} R_{2}^{-1} D_{2}^{\top} & 2 \mu D_{2} R_{2}^{-1} D_{2}^{\top}
\end{array}\right), \\
& \bar{Q}_{1}=\frac{\left(\begin{array}{cc}
1 \\
Q_{1}+2 \mu Q_{2} & -Q_{2} \\
-Q_{2} & 2 \lambda Q_{2}
\end{array}\right),}{1+4 \lambda \mu}\left(\begin{array}{cc}
\Phi_{1}+2 \mu \Phi_{2} & -\Phi_{2} \\
-\Phi_{2} & 2 \lambda \Phi_{2}
\end{array}\right) .
\end{aligned}
$$

Now it is easy to check that $\overline{\mathcal{B}}_{1}, \overline{\mathcal{B}}_{2}, \overline{\mathcal{D}}_{1}, \overline{\mathcal{D}}_{2}, \bar{Q}_{1}$ and $\bar{\Phi}_{1}$ are symmetric and positive definite. Repeating the approach above, we can derive a standard backward stochastic Riccati equation as follows:

$$
\begin{aligned}
\mathrm{d} \overline{\mathcal{R}}(t)= & {\left[\overline{\mathcal{A}}(t) \overline{\mathcal{R}}(t)+\overline{\mathcal{C}}^{\top}(t) \bar{\Xi}(t)+\bar{Q}_{1}(t)\right.} \\
& +\overline{\mathcal{R}}(t)\left[\overline{\mathcal{A}}(t)-\overline{\mathcal{B}}_{1}(t) \overline{\mathcal{R}}(t)-\overline{\mathcal{B}}_{2}(t) \bar{\Xi}(t)\right] \\
& \left.+\bar{\Psi}(t)\left[\overline{\mathcal{C}}(t)-\overline{\mathcal{D}}_{1}(t) \overline{\mathcal{R}}(t)-\overline{\mathcal{D}}_{2}(t) \bar{\Xi}(t)\right]\right] \mathrm{d} t \\
& -\bar{\Psi}(t) \mathrm{d} W(t) \\
\overline{\mathcal{R}}(T)= & \bar{\Phi}_{1} .
\end{aligned}
$$

where

$$
\Xi(t)=\left(I+\overline{\mathcal{R}}(t) \overline{\mathcal{D}}_{2}(t)\right)^{-1}\left[\overline{\mathcal{R}}(t) \overline{\mathcal{C}}(t)-\overline{\mathcal{R}}(t) \overline{\mathcal{D}}_{1}(t) \overline{\mathcal{R}}(t)+\bar{\Psi}(t)\right] .
$$

From Tang [22], we know that the Riccati equation (37) admits a unique solution. Moreover, we have

$$
\begin{aligned}
\bar{P} & =\overline{\mathcal{R}} \bar{X}, \\
\bar{Q} & =\bar{\Xi} \bar{X}, \\
\mathcal{R} & =\Upsilon \overline{\mathcal{R}}, \\
\Psi & =\Upsilon \bar{\Psi} .
\end{aligned}
$$

From the fact

$$
\begin{aligned}
P & =\Upsilon \bar{P}=\Upsilon \overline{\mathcal{R}} \bar{X}=\Upsilon \overline{\mathcal{R}} X, \\
Q & =\Upsilon \bar{Q}=\Upsilon \bar{\Xi} \bar{X}=\Upsilon \bar{\Xi} X,
\end{aligned}
$$

we state that the AOL Stackelberg solution $\left(u^{*}(\cdot), v^{*}(\cdot)\right)$ presents a feedback representation with respect to state $(x, k)$. The proof is completed. 


\subsection{The ACLM fashion}

In this subsection, we aforehand suppose that the derivative $\frac{\partial u}{\partial x}$ to be bounded. As we shall see that the derivative enters into the coefficient of the adjoint equation, its boundedness ensures the well-posedness of the leader's problem whenever affine strategies are considered. For simplicity, we study a one-dimensional linear quadratic game, with the state equation as follows:

$$
\left\{\begin{aligned}
\mathrm{d} x & =\left[A x+B_{1} u+B_{2} v\right] \mathrm{d} t+\left[C x+D_{1} u+D_{2} v\right] \mathrm{d} W(t) \\
x(0) & =x_{0} \in \mathbb{R} .
\end{aligned}\right.
$$

The cost functionals for the leader and the follower to minimize are given, respectively, as follows:

$$
\mathcal{J}_{1}(u, v)=\frac{1}{2} \mathbb{E}\left[\int_{0}^{T}\left[\left(Q_{1} x^{2}+R_{1} u^{2}\right)\right] \mathrm{d} t+\Phi_{1} x^{2}(T)\right]
$$

and

$$
\mathcal{J}_{2}(u, v)=\frac{1}{2} \mathbb{E}\left[\int_{0}^{T}\left(Q_{2} x^{2}+R_{2} v^{2}\right) \mathrm{d} t+\Phi_{2} x^{2}(T)\right] .
$$

The admissible strategy spaces for the leader and the follower are denoted by

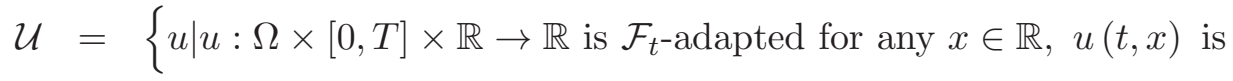

$$
\begin{aligned}
& \text { continuously differentiable in } \left.x \text { for any }(t, x) \in \Omega \times[0, T] \text { satisfying }\left|\frac{\partial u}{\partial x}\right|<K\right\} \text {, } \\
& \mathcal{V}=\left\{v \mid: \Omega \times[0, T] \times \mathbb{R} \times \mathcal{U} \rightarrow \Gamma_{2} \text { is } \mathcal{F}_{t} \text {-adapted for any } x \in \mathbb{R} \text { and } u \in \mathcal{U}\right\} .
\end{aligned}
$$

For any given $u \in \mathcal{U}$, the follower responses a unique optimal strategy $v^{*} \in \mathcal{V}$. From (10), we get

$$
v^{*}(u)=\mathbf{P}_{\Gamma_{2}}\left[-R_{2}^{-1}\left(B_{2}^{\top} p_{2}+D_{2}^{\top} q_{2}\right)\right]
$$

where $p_{2}$ and $q_{2}$ satisfy

$$
\left\{\begin{aligned}
-\mathrm{d} p_{2} & =\left[\left(A+B_{1} \frac{\partial u}{\partial x}\right) p_{2}+\left(C+D_{1} \frac{\partial u}{\partial x}\right) q_{2}+Q_{2} x\right] \mathrm{d} t-q_{2} \mathrm{~d} W(t) \\
p_{2}(T) & =\Phi_{2} x(T) .
\end{aligned}\right.
$$

Now we formulate the leader's optimal control problem:

$$
\min _{u \in \mathcal{U}} \mathcal{J}_{1}\left(u, v^{*}\right)
$$

subject to

$$
\left\{\begin{aligned}
\mathrm{d} x= & {\left[A x+B_{1} u-B_{2} \mathbf{P}_{\Gamma_{2}}\left[R_{2}^{-1}\left(B_{2}^{\top} p_{2}+D_{2}^{\top} q_{2}\right)\right]\right] \mathrm{d} t } \\
& +\left[C x+D_{1} u-D_{2} \mathbf{P}_{\Gamma_{2}}\left[R_{2}^{-1}\left(B_{2}^{\top} p_{2}+D_{2}^{\top} q_{2}\right)\right]\right] \mathrm{d} W(t) \\
-\mathrm{d} p_{2}= & {\left[\left(A+B_{1} \frac{\partial u}{\partial x}\right) p_{2}+\left(C+D_{1} \frac{\partial u}{\partial x}\right) q_{2}+Q_{2} x\right] \mathrm{d} t-q_{2} \mathrm{~d} W(t) } \\
p_{2}(T)= & \Phi_{2} x(T)
\end{aligned}\right.
$$

Note that FBSDEs (42) are fully coupled. Due to the boundedness of $\frac{\partial u}{\partial x}$, (42) admit a unique solution. Hence the leader's problem is well-posed. By means of Theorem 2.1, the leader can select his strategy among affine functions

$$
u(t, x)=u_{2}(t) x+u_{1}(t),
$$

where $u_{2}$ and $u_{1}$ are adapted processes with $\left|u_{2}\right| \leq K$. Thus, the leader's problem can be described as follows: The state equation is

$$
\left\{\begin{aligned}
\mathrm{d} x= & {\left[\left(A+B_{1} u_{2}\right) x+B_{1} u_{1}-B_{2} \mathbf{P}_{\Gamma_{2}}\left[R_{2}^{-1}\left(B_{2}^{\top} p_{2}+D_{2}^{\top} q_{2}\right)\right]\right] \mathrm{d} t } \\
& +\left[\left(C+D_{1} u_{2}\right) x+D_{1} u_{1}-D_{2} \mathbf{P}_{\Gamma_{2}}\left[R_{2}^{-1}\left(B_{2}^{\top} p_{2}+D_{2}^{\top} q_{2}\right)\right]\right] \mathrm{d} W(t), \\
-\mathrm{d} p_{2}= & {\left[\left(A+B_{1} u_{2}\right) p_{2}+\left(C+D_{1} u_{2}\right) q_{2}+Q_{2} x\right] \mathrm{d} t-q_{2} \mathrm{~d} W(t), } \\
p_{2}(T)= & \Phi_{2} x(T) .
\end{aligned}\right.
$$


The cost functional to be minimized:

$$
\min _{u \in \mathcal{U}} \mathcal{J}_{1}(u, v)=\min _{u \in \mathcal{U}} \frac{1}{2} \mathbb{E}\left[\int_{0}^{T}\left[\left(Q_{1} x^{2}+R_{1}\left(u_{2} x+u_{1}\right)^{2}\right)\right] \mathrm{d} t+\Phi_{1} x^{2}(T)\right] .
$$

For any $\left(u_{1}, u_{2}\right)$, we can get the existence and uniqueness of the solution of (43) by the monotonicity condition (Proposition A.2). Therefore, the leader's problem with strategies restricted to be of affine form is well-posed. Now we apply the maximum principle to obtain the Hamiltonian system and the related Riccati equation for the leader's problem (43)-(44).

Set

$$
\begin{aligned}
& \mathcal{H}_{3}\left(t, u_{1}, u_{2}, x, \chi, p_{1}, q_{1}, p_{2}, q_{2}\right) \\
= & p_{1}\left[\left(A+B_{1} u_{2}\right) x+B_{1} u_{1}-B_{2} \mathbf{P}_{\Gamma_{2}}\left[R_{2}^{-1}\left(B_{2}^{\top} p_{2}+D_{2}^{\top} q_{2}\right)\right]\right] \\
& +q_{1}\left[\left(C+D_{1} u_{2}\right) x+D_{1} u_{1}-D_{2} \mathbf{P}_{\Gamma_{2}}\left[R_{2}^{-1}\left(B_{2}^{\top} p_{2}+D_{2}^{\top} q_{2}\right)\right]\right] \\
& -\chi\left[\left(A+B_{1} u_{2}\right) p_{2}+\left(C+D_{1} u_{2}\right) q_{2}+Q_{2} x\right] \\
& +\frac{1}{2} Q_{1} x^{2}+\frac{1}{2} R_{1}\left(u_{2} x+u_{1}\right)^{2} .
\end{aligned}
$$

Clearly, $\mathcal{H}_{3}$ is quadratic with respect to $u_{1}$. Thus fix $u_{2}$ first, the minimizer of $\mathcal{H}_{3}$ can be obtained

$$
u_{1}^{*}=-u_{2} x-R_{1}^{-1} p_{1} B_{1}-R_{1}^{-1} q_{1} D_{1} .
$$

Inserting (46) into (45), we can see that the only term involving $u_{2}$ is $-\chi\left(B_{1} p_{2}+D_{1} q_{2}\right) u_{2}$. Hence, the optimal $u_{2}$ can be attained by

$$
u_{2}^{*}=\operatorname{sgn}\left(\chi\left(B_{1} p_{2}+D_{1} q_{2}\right)\right) K,
$$

where

$$
\operatorname{sgn}(x)=\left\{\begin{array}{cc}
1 & \text { if } x>0 \\
0 & \text { if } x=0 \\
-1 & \text { if } x<0
\end{array}\right.
$$

Now for simplicity, let $\Gamma_{2}=\mathbb{R}$. In this case,

$$
\begin{aligned}
& \mathcal{H}_{3}\left(t, u_{1}, u_{2}, x, \chi, p_{1}, q_{1}, p_{2}, q_{2}\right) \\
= & \left.p_{1}\left[\left(A+B_{1} u_{2}\right) x+B_{1} u_{1}-B_{2} R_{2}^{-1}\left(B_{2}^{\top} p_{2}+D_{2}^{\top} q_{2}\right)\right]\right] \\
& +q_{1}\left[\left(C+D_{1} u_{2}\right) x+D_{1} u_{1}-D_{2} R_{2}^{-1}\left(B_{2}^{\top} p_{2}+D_{2}^{\top} q_{2}\right)\right] \\
& -\chi\left[\left(A+B_{1} u_{2}\right) p_{2}+\left(C+D_{1} u_{2}\right) q_{2}+Q_{2} x\right] \\
& +\frac{1}{2} Q_{1} x^{2}+\frac{1}{2} R_{1}\left(u_{2} x+u_{1}\right)^{2} .
\end{aligned}
$$

So

$$
\left\{\begin{aligned}
\mathrm{d} x= & {\left[\left(A+B_{1} u_{2}^{*}\right) x+B_{1} u_{1}^{*}-B_{2} R_{2}^{-1}\left(B_{2} p_{2}+D_{2}^{\top} q_{2}\right)\right] \mathrm{d} t } \\
& +\left[\left(C+D_{1} u_{2}^{*}\right) x+D_{1} u_{1}^{*}-D_{2} R_{2}^{-1}\left(B_{2}^{\top} p_{2}+D_{2}^{\top} q_{2}\right)\right] \mathrm{d} W(t) \\
\mathrm{d} \chi= & {\left[\left(A+B_{1} u_{2}^{*}\right) \chi+B_{2} R_{2}^{-1} B_{2}^{\top} p_{1}+D_{2} R_{2}^{-1} B_{2}^{\top} q_{1}\right] \mathrm{d} t } \\
& +\left[\left(C+D_{1} u_{2}^{*}\right) \chi+B_{2} R_{2}^{-1} D_{2}^{\top} p_{1}+D_{2} R_{2}^{-1} D_{2}^{\top} q_{1}\right] \mathrm{d} W(t) \\
-\mathrm{d} p_{1}= & {\left[\left(A+B_{1} u_{2}^{*}\right) p_{1}+\left(C+D_{1} u_{2}^{*}\right) q_{1}-\chi Q_{2}+Q_{1} x+R_{1}\left(u_{2}^{*} x+u_{1}^{*}\right) u_{2}^{*}\right] \mathrm{d} t } \\
& -q_{1}(t) \mathrm{d} W(t) \\
x(0)= & x_{0}, \chi(0)=0, p_{1}(T)=-\Phi_{2} \chi(T)+\Phi_{1} x(T) .
\end{aligned}\right.
$$


Put (46) and (47) into above, we get

$$
\left\{\begin{aligned}
\mathrm{d} x= & {\left[A x-B_{1}^{2} R_{1}^{-1}\left(B_{1} p_{1}+D_{1} q_{1}\right)-B_{2} R_{2}^{-1}\left(B_{2} p_{2}+D_{2}^{\top} q_{2}\right)\right] \mathrm{d} t } \\
& +\left[C x-D_{1}^{2} R_{1}^{-1}\left(B_{1} p_{1}+D_{1} q_{1}\right)-D_{2} R_{2}^{-1}\left(B_{2} p_{2}+D_{2}^{\top} q_{2}\right)\right] \mathrm{d} W(t) \\
\mathrm{d} \chi= & {\left[A \chi+B_{1} \operatorname{sgn}\left(\chi\left(B_{1} p_{2}+D_{1} q_{2}\right)\right) K \chi+B_{2} R_{2}^{-1} B_{2}^{\top} p_{1}+D_{2} R_{2}^{-1} B_{2}^{\top} q_{1}\right] \mathrm{d} t } \\
& +\left[C \chi+D_{1} \operatorname{sgn}\left(\chi\left(B_{1} p_{2}+D_{1} q_{2}\right)\right) K \chi+B_{2} R_{2}^{-1} D_{2}^{\top} p_{1}+D_{2} R_{2}^{-1} D_{2}^{\top} q_{1}\right] \mathrm{d} W(t), \\
-\mathrm{d} p_{1}= & {\left[A p_{1}+C q_{1}-\chi Q_{2}+Q_{1} x\right] \mathrm{d} t-q_{1}(t) \mathrm{d} W(t), } \\
-\mathrm{d} p_{2}= & {\left[\left(A+B_{1} \operatorname{sgn}\left(\chi\left(B_{1} p_{2}+D_{1} q_{2}\right)\right) K\right) p_{2}\right.} \\
& \left.+\left(C+D_{1} \operatorname{sgn}\left(\chi\left(B_{1} p_{2}+D_{1} q_{2}\right)\right) K\right) q_{2}+Q_{2} x\right] \mathrm{d} t-q_{2} \mathrm{~d} W(t), \\
x(0)= & x_{0}, \chi(0)=0, p_{1}(T)=-\Phi_{2} \chi(T)+\Phi_{1} x(T), p_{2}(T)=\Phi_{2} x(T) .
\end{aligned}\right.
$$

Clearly, the Hamilton system (49) is highly complex due to the nonlinear term $\operatorname{sgn}\left(\chi\left(B_{1} p_{2}+D_{1} q_{2}\right)\right)$. Specifically, the state $(x, \chi)^{\top}$ cannot be written as a linear equation with respect to $\left(p_{1}, p_{2}\right)^{\top}$. However, $\operatorname{sgn}\left(\chi\left(B_{1} p_{2}+D_{1} q_{2}\right)\right)$ doesn't appear into $x$, besides, the terminal condition $p_{1}(T)$, $p_{2}(T)$ contain $x(T)$. Hence, we only regard $x$ as the state and postulate

$$
\begin{aligned}
\chi(t) & =\alpha(t) x(t), \\
p_{1}(t) & =\beta(t) x(t), \\
p_{2}(t) & =\gamma(t) x(t),
\end{aligned}
$$

where

$$
\begin{aligned}
& \mathrm{d} \alpha(t)=\alpha_{1}(t) \mathrm{d} t+\alpha_{2}(t) \mathrm{d} W(t), \\
& \mathrm{d} \beta(t)=\beta_{1}(t) \mathrm{d} t+\beta_{2}(t) \mathrm{d} W(t), \\
& \mathrm{d} \gamma(t)=\gamma_{1}(t) \mathrm{d} t+\gamma_{2}(t) \mathrm{d} W(t) .
\end{aligned}
$$

We first look for $q_{1}, q_{2}$. Applying Itô's formula to $\beta(t) x(t)$ and $\gamma(t) x(t)$, we have

$$
\begin{aligned}
& \beta\left[A x-B_{1}^{2} R_{1}^{-1}\left(B_{1} \beta x+D_{1} q_{1}\right)-B_{2} R_{2}^{-1}\left(B_{2} \gamma x+D_{2}^{\top} q_{2}\right)\right] \mathrm{d} t \\
& +\beta\left[C x-D_{1}^{2} R_{1}^{-1}\left(B_{1} \beta x+D_{1} q_{1}\right)-D_{2} R_{2}^{-1}\left(B_{2} \gamma x+D_{2}^{\top} q_{2}\right)\right] \mathrm{d} W(t) \\
& +x \beta_{1} \mathrm{~d} t+x \beta_{2} \mathrm{~d} W(t) \\
& +\beta_{2}\left[C x-D_{1}^{2} R_{1}^{-1}\left(B_{1} \beta x+D_{1} q_{1}\right)-D_{2} R_{2}^{-1}\left(B_{2} \gamma x+D_{2}^{\top} q_{2}\right)\right] \mathrm{d} t \\
= & \mathrm{d} p_{1} \\
= & -\left[A \beta x+C q_{1}-\alpha x Q_{2}+Q_{1} x\right] \mathrm{d} t+q_{1} \mathrm{~d} W(t),
\end{aligned}
$$

and

$$
\begin{aligned}
& \gamma\left[A x-B_{1}^{2} R_{1}^{-1}\left(B_{1} \beta x+D_{1} q_{1}\right)-B_{2} R_{2}^{-1}\left(B_{2} \gamma x+D_{2}^{\top} q_{2}\right)\right] \mathrm{d} t \\
& +\gamma\left[C x-D_{1}^{2} R_{1}^{-1}\left(B_{1} \beta x+D_{1} q_{1}\right)-D_{2} R_{2}^{-1}\left(B_{2} \gamma x+D_{2}^{\top} q_{2}\right)\right] \mathrm{d} W(t) \\
& +x \gamma_{1} \mathrm{~d} t+x \gamma_{2} \mathrm{~d} W(t) \\
= & \mathrm{d} p_{2} \\
= & -\left[\left(A+B_{1} \operatorname{sgn}\left(\alpha x\left(B_{1} p_{2}+D_{1} q_{2}\right)\right) K\right) p_{2}\right. \\
& \left.+\left(C+D_{1} \operatorname{sgn}\left(\alpha x\left(B_{1} p_{2}+D_{1} q_{2}\right)\right) K\right) q_{2}+Q_{2} x\right] \mathrm{d} t+q_{2} \mathrm{~d} W(t),
\end{aligned}
$$

So

$$
\begin{aligned}
q_{1}= & \beta C x-\beta D_{1}^{2} R_{1}^{-1} B_{1} \beta x-\beta D_{1}^{2} R_{1}^{-1} D_{1} q_{1} \\
& -\beta D_{2} R_{2}^{-1} B_{2} \gamma x-\beta D_{2} R_{2}^{-1} D_{2}^{\top} q_{2}+x \beta_{2}, \\
q_{2}= & \gamma C x-\gamma D_{1}^{2} R_{1}^{-1} B_{1} \beta x-\gamma D_{1}^{2} R_{1}^{-1} D_{1} q_{1} \\
& -\gamma D_{2} R_{2}^{-1} B_{2} \gamma x-\gamma D_{2} R_{2}^{-1} D_{2}^{\top} q_{2}+x \gamma_{2},
\end{aligned}
$$


namely,

$$
\left\{\begin{array}{l}
\left(1+\beta D_{1}^{2} R_{1}^{-1} D_{1}\right) q_{1}+\beta D_{2} R_{2}^{-1} D_{2}^{\top} q_{2}=\Xi_{1} x \\
\gamma D_{1}^{2} R_{1}^{-1} D_{1} q_{1}+\left(1+\gamma D_{2} R_{2}^{-1} D_{2}^{\top}\right) q_{2}=\Xi_{2} x
\end{array}\right.
$$

where

$$
\begin{aligned}
& \Xi_{1}=\beta C-\beta D_{1}^{2} R_{1}^{-1} B_{1} \beta-\beta D_{2} R_{2}^{-1} B_{2} \gamma+\beta_{2}, \\
& \Xi_{2}=\gamma C-\gamma D_{1}^{2} R_{1}^{-1} B_{1} \beta-\gamma D_{2} R_{2}^{-1} B_{2} \gamma+\gamma_{2} .
\end{aligned}
$$

It is easy to get

$$
\begin{aligned}
& q_{1}=\Delta_{1} x, \\
& q_{2}=\Delta_{2} x,
\end{aligned}
$$

where

$$
\begin{aligned}
\Delta_{1} & =\frac{\left[\Xi_{1}\left(1+\gamma D_{2} R_{2}^{-1} D_{2}^{\top}\right)-\Xi_{2} \beta D_{2} R_{2}^{-1} D_{2}^{\top}\right]}{\left(1+\beta D_{1}^{2} R_{1}^{-1} D_{1}\right)\left(1+\gamma D_{2} R_{2}^{-1} D_{2}^{\top}\right)-\beta D_{2} R_{2}^{-1} D_{2}^{\top} \gamma D_{1}^{2} R_{1}^{-1} D_{1}}, \\
\Delta_{2} & =\frac{\left[\Xi_{2}\left(1+\beta D_{1}^{2} R_{1}^{-1} D_{1}\right)-\Xi_{1} \gamma D_{1}^{2} R_{1}^{-1} D_{1}\right]}{\left(1+\beta D_{1}^{2} R_{1}^{-1} D_{1}\right)\left(1+\gamma D_{2} R_{2}^{-1} D_{2}^{\top}\right)-\beta D_{2} R_{2}^{-1} D_{2}^{\top} \gamma D_{1}^{2} R_{1}^{-1} D_{1}} .
\end{aligned}
$$

Moreover,

$$
\begin{aligned}
\beta_{1}= & -\left[A \beta+C \Delta_{1}-\alpha Q_{2}+Q_{1}\right] \\
& -\beta\left[A-B_{1}^{2} R_{1}^{-1}\left(B_{1} \beta+D_{1} \Delta_{1}\right)-B_{2} R_{2}^{-1}\left(B_{2} \gamma+D_{2}^{\top} \Delta_{2}\right)\right] \\
& -\beta_{2}\left[C-D_{1}^{2} R_{1}^{-1}\left(B_{1} \beta+D_{1} \Delta_{1}\right)-D_{2} R_{2}^{-1}\left(B_{2} \gamma+D_{2}^{\top} \Delta_{2}\right)\right]
\end{aligned}
$$

and

$$
\begin{aligned}
\gamma_{1}= & -\left[\left(A+B_{1} \operatorname{sgn}\left(\alpha\left(B_{1} \gamma+D_{1} \Delta_{2}\right)\right) K\right) \gamma\right. \\
& \left.+\left(C+D_{1} \operatorname{sgn}\left(\alpha\left(B_{1} \gamma+D_{1} \Delta_{2}\right)\right) K\right) \Delta_{2}+Q_{2}\right] \\
& -\gamma\left[A-B_{1}^{2} R_{1}^{-1}\left(B_{1} \beta+D_{1} \Delta_{1}\right)-B_{2} R_{2}^{-1}\left(B_{2} \gamma+D_{2}^{\top} \Delta_{2}\right)\right] .
\end{aligned}
$$

Repeating the method used above, we have

$$
\begin{aligned}
& \alpha\left[A x-B_{1}^{2} R_{1}^{-1}\left(B_{1} \beta x+D_{1} q_{1}\right)-B_{2} R_{2}^{-1}\left(B_{2} \gamma x+D_{2}^{\top} \rho_{2} x\right)\right] \mathrm{d} t+x \alpha_{1} \mathrm{~d} t+x \alpha_{2} \mathrm{~d} W \\
& +\alpha\left[C x-D_{1}^{2} R_{1}^{-1}\left(B_{1} \beta x+D_{1} q_{1}\right)-D_{2} R_{2}^{-1}\left(B_{2} \gamma x+D_{2}^{\top} q_{2}\right)\right] \mathrm{d} W(t) \\
& +\alpha_{2}\left[C x-D_{1}^{2} R_{1}^{-1}\left(B_{1} \beta x+D_{1} q_{1}\right)-D_{2} R_{2}^{-1}\left(B_{2} \gamma x+D_{2}^{\top} q_{2}\right)\right] \mathrm{d} t \\
= & \mathrm{d} \chi \\
= & {\left[A \alpha x+B_{1} \operatorname{sgn}\left(\alpha x\left(B_{1} \gamma x+D_{1} q_{2}\right)\right) K \alpha x+B_{2} R_{2}^{-1} B_{2}^{\top} \beta x+D_{2} R_{2}^{-1} B_{2}^{\top} q_{1}\right] \mathrm{d} t } \\
& +\left[C \alpha x+D_{1} \operatorname{sgn}\left(\alpha x\left(B_{1} \gamma x+D_{1} q_{2}\right)\right) K \alpha x+B_{2} R_{2}^{-1} D_{2}^{\top} \beta x+D_{2} R_{2}^{-1} D_{2}^{\top} q_{1}\right] \mathrm{d} W(t) .
\end{aligned}
$$

By comparing (49) and (50), we have

$$
\begin{aligned}
\alpha_{2}= & \alpha\left[D_{1}^{2} R_{1}^{-1}\left(B_{1} \beta+D_{1} \Delta_{1}\right)+D_{2} R_{2}^{-1}\left(B_{2} \gamma+D_{2}^{\top} \Delta_{2}\right)\right] \\
& +D_{1} \operatorname{sgn}\left(\alpha\left(B_{1} \gamma+D_{1} \Delta_{2}\right)\right) \alpha+B_{2} R_{2}^{-1} D_{2}^{\top} \beta+D_{2} R_{2}^{-1} D_{2}^{\top} \Delta_{1}, \\
\alpha_{1}= & B_{1} \operatorname{sgn}\left(\alpha\left(B_{1} \gamma+D_{1} \Delta_{2}\right)\right) K \alpha+B_{2} R_{2}^{-1} B_{2}^{\top} \beta+D_{2} R_{2}^{-1} B_{2}^{\top} \Delta_{1} \\
& +\alpha\left[B_{1}^{2} R_{1}^{-1}\left(B_{1} \beta+D_{1} \Delta_{1}\right)+B_{2} R_{2}^{-1}\left(B_{2} \gamma+D_{2}^{\top} \Delta_{2}\right)\right] \\
& +\alpha_{2}\left[C+D_{1}^{2} R_{1}^{-1}\left(B_{1} \beta+D_{1} \Delta_{1}\right)+D_{2} R_{2}^{-1}\left(B_{2} \gamma+D_{2}^{\top} \Delta_{2}\right)\right] .
\end{aligned}
$$


Now we are able to announce a new system as follows:

$$
\begin{aligned}
\mathrm{d} x^{*}= & {\left[A-B_{1}^{2} R_{1}^{-1}\left(B_{1} \beta+D_{1} \Delta_{1}\right)-B_{2} R_{2}^{-1}\left(B_{2} \gamma+D_{2}^{\top} \Delta_{2}\right)\right] x^{*} \mathrm{~d} t } \\
& +\left[C-D_{1}^{2} R_{1}^{-1}\left(B_{1} \beta+D_{1} \Delta_{1}\right)-D_{2} R_{2}^{-1}\left(B_{2} \gamma+D_{2}^{\top} \Delta_{2}\right)\right] x^{*} \mathrm{~d} W(t), \\
\mathrm{d} \alpha= & {\left[B_{1} \operatorname{sgn}\left(\alpha\left(B_{1} \gamma+D_{1} \Delta_{2}\right)\right) K \alpha+B_{2} R_{2}^{-1} B_{2}^{\top} \beta+D_{2} R_{2}^{-1} B_{2}^{\top} \Delta_{1}\right.} \\
& +\alpha\left[B_{1}^{2} R_{1}^{-1}\left(B_{1} \beta+D_{1} \Delta_{1}\right)+B_{2} R_{2}^{-1}\left(B_{2} \gamma+D_{2}^{\top} \Delta_{2}\right)\right] \\
& \left.+\alpha_{2}\left[C+D_{1}^{2} R_{1}^{-1}\left(B_{1} \beta+D_{1} \Delta_{1}\right)+D_{2} R_{2}^{-1}\left(B_{2} \gamma+D_{2}^{\top} \Delta_{2}\right)\right]\right] \mathrm{d} t \\
& {\left[\alpha\left[D_{1}^{2} R_{1}^{-1}\left(B_{1} \beta+D_{1} \Delta_{1}\right)+D_{2} R_{2}^{-1}\left(B_{2} \gamma+D_{2}^{\top} \Delta_{2}\right)\right]\right.} \\
& \left.+D_{1} \operatorname{sgn}\left(\alpha\left(B_{1} \gamma+D_{1} \Delta_{2}\right)\right) K \alpha+B_{2} R_{2}^{-1} D_{2}^{\top} \beta+D_{2} R_{2}^{-1} D_{2}^{\top} \Delta_{1}\right] \mathrm{d} W(t), \\
\mathrm{d} \beta= & \left\{-\left[A \beta+C \Delta_{1}-\alpha Q_{2}+Q_{1}\right]\right. \\
& -\beta\left[A-B_{1}^{2} R_{1}^{-1}\left(B_{1} \beta+D_{1} \Delta_{1}\right)-B_{2} R_{2}^{-1}\left(B_{2} \gamma+D_{2}^{\top} \Delta_{2}\right)\right] \\
& \left.-\beta_{2}\left[C-D_{1}^{2} R_{1}^{-1}\left(B_{1} \beta+D_{1} \Delta_{1}\right)-D_{2} R_{2}^{-1}\left(B_{2} \gamma+D_{2}^{\top} \Delta_{2}\right)\right]\right\} \mathrm{d} t+\beta_{2} \mathrm{~d} W(t), \\
\mathrm{d} \gamma= & \left\{-\left[\left(A+B_{1} \operatorname{sgn}\left(\alpha\left(B_{1} \gamma+D_{1} \Delta_{2}\right)\right) K^{\prime} \gamma\right.\right.\right. \\
& \left.+\left(C+D_{1} \operatorname{sgn}\left(\alpha\left(B_{1} \gamma+D_{1} \Delta_{2}\right)\right) K\right) \Delta_{2}+Q_{2}\right] \\
& \left.-\gamma\left[A-B_{1}^{2} R_{1}^{-1}\left(B_{1} \beta+D_{1} \Delta_{1}\right)-B_{2} R_{2}^{-1}\left(B_{2} \gamma+D_{2}^{\top} \Delta_{2}\right)\right]\right\} \mathrm{d} t+\gamma_{2} \mathrm{~d} W(t), \\
\alpha(0)= & , \beta(T)=-\Phi_{2} \alpha(T)+\Phi_{1}, \gamma(T)=\Phi_{2} .
\end{aligned}
$$

Suppose that FBSDEs (53) admit a unique solution, denoted by $\left(x^{*}, \alpha, \beta, \gamma, \beta_{2}, \gamma_{2}\right)$, which actually solve the Hamiltonian system (49). As a result, a candidate for the leader's optimal strategy can be expressed as

$$
\begin{aligned}
u(t, x)= & \operatorname{sgn}\left(\alpha\left(B_{1} \gamma+D_{1} \Delta_{2}\right)\right) K x-\operatorname{sgn}\left(\alpha\left(B_{1} \gamma+D_{1} \Delta_{2}\right)\right) K x^{*}(t) \\
& -R_{1}^{-1} \beta B_{1} x^{*}(t)-R_{1}^{-1} \Delta_{1} D_{1} x^{*}(t) .
\end{aligned}
$$

Remark 3.3 From [54), it indicates that, comparing with Bensoussan et al. [18], the matrices $D_{1}, D_{2}$ will impose on $u(t, x)$ via $D_{1} \Delta_{2}$ and $\Delta_{1} D_{1}$. If $D_{1}=D_{2}=0$, 54) reduces to Bensoussan et al.'s type (see [18]).

\section{Conclusions and remarks}

In this paper, we are concerned on the solutions of stochastic Stackelberg differential games within two information structures: AOL and ACLM patterns under convex control domain. Having maximum principle for the former kind of game as a basis, we give the necessary conditions for the leader's optimal strategy in the latter game. To illustrate our theoretic results, we study the LQ stochastic Stackelberg differential games. For AOL case, on the one hand, we prove the existence and uniqueness of Hamiltonian system of leader's with projection operator and derive a kind of standard backward stochastic Riccati equation. For ACLM case, we also give a Riccati equation with non-linear term and then provide the leader's optimal strategy.

There are some topics deserved to study displaying in the following: (i) The Riccati equation derived in the framework of ACLM case consists of complex coupled FBSDEs with non-linear term. The general conditions to guarantee the existence and uniqueness is not known. (ii) As observed that the control set is limited to convex, a natural question arises: How to establish the maximum principle for general case, namely, non-convex control domain? Certainly, the second-order adjoint equation is employed, which makes the system extremely complicated. The Hamiltonian system in this situation actually involves six types of (forward or backward) stochastic differential equations. (iii) It is necessary to point out that the Stackelberg game considered in this paper is limited to in the complete information background. In other word, both the leader and the follower can observe the state process of the stochastic system directly, which, however, is impossible in reality. As a matter of fact, both of them can only announce partial information because of the market competition, information-delay, private information 
and limitation policy by the government, etc. Therefore, it is necessary to study the Stackelberg game with partial and asymmetric information (see [32, 33]). These possible extensions to the Stackelberg stochastic differential game no doubt promise to be interesting research directions. We shall response these challenging topics in our future work.

\section{A Properties of projection $\mathrm{P}_{\Gamma}$}

For the readers' convenience, let us recall the following properties of projection $\mathbf{P}_{\Gamma}$ onto a closed convex set, see [34], Chapter 5.

Proposition A.1 For a nonempty closed convex set $\Gamma \subset \mathbb{R}^{m}$, for every $x \in \mathbb{R}^{m}$, there exists a unique $x^{*} \in \Gamma$, such that

$$
\left|x-x^{*}\right|=\min _{y \in \Gamma}|x-y|=: \operatorname{dist}(x, \Gamma) .
$$

Moreover, $x^{*}$ is characterized by the property

$$
x^{*} \in \Gamma, \quad\left\langle x^{*}-x, x^{*}-y\right\rangle \leq 0 \quad \forall y \in \Gamma .
$$

The above element $x^{*}$ is called the projection of $x$ onto $\Gamma$ and is denoted by $\mathbf{P}_{\Gamma}[x]$.

From above theorem, it is easy to show that

Proposition A.2 Let $\Gamma \subset \mathbb{R}^{m}$ be a nonempty closed convex set, then we have

$$
\left|\mathbf{P}_{\Gamma}[x]-\mathbf{P}_{\Gamma}[y]\right|^{2} \leq\left\langle\mathbf{P}_{\Gamma}[x]-\mathbf{P}_{\Gamma}[y], x-y\right\rangle .
$$

Proposition A.3 Let $\Gamma \subset \mathbb{R}^{m}$ be a nonempty closed convex set, then the projection $\mathbf{P}_{\Gamma}$ does not increase the distance, i.e.

$$
\left|\mathbf{P}_{\Gamma}[x]-\mathbf{P}_{\Gamma}[y]\right| \leq|x-y|
$$

Now let us consider $\mathbb{R}^{m}$ and the projection $\mathbf{P}_{\Gamma}$ both with the norm $\|\cdot\|_{R_{0}}:=\left\langle R_{0}^{\frac{1}{2}} \cdot, R_{0}^{\frac{1}{2}} \cdot\right\rangle$, from (56), we have

Proposition A.4 Let $\Gamma \subset \mathbb{R}^{m}$ be a nonempty closed convex set, then

$$
\left\langle\left\langle\mathbf{P}_{\Gamma}[x]-\mathbf{P}_{\Gamma}[y], x-y\right\rangle\right\rangle=\left\langle R^{\frac{1}{2}}\left(\mathbf{P}_{\Gamma}[x]-\mathbf{P}_{\Gamma}[y]\right), R^{\frac{1}{2}}(x-y)\right\rangle \geq 0 .
$$

\section{B The proof of Theorem 3.1}

Proof. (Uniqueness) Suppose that there exists two solutions: $\left(x^{1}, p_{2}^{1}, q_{2}^{1}\right),\left(x^{2}, p_{2}^{2}, q_{2}^{2}\right)$ and denote

$$
\hat{x}=x^{1}-x^{2}, \quad \hat{p}_{2}=p_{2}^{1}-p_{2}^{2}, \quad \hat{q}_{2}=q_{2}^{1}-q_{2}^{2} .
$$

Then, we have

$$
\left\{\begin{aligned}
\mathrm{d} \hat{x}(t)= & {\left[A(t) \hat{x}(t)+B_{2}(t) \hat{\varphi}_{2}\left(t, \hat{p}_{2}(t), \hat{q}_{2}(t)\right)\right] \mathrm{d} t } \\
& +\left[C(t) \hat{x}(t)+D_{2}(t) \hat{\varphi}_{2}\left(t, \hat{p}_{2}(t), \hat{q}_{2}(t)\right)\right] \mathrm{d} W(t) \\
-\mathrm{d} \hat{p}_{2}(t)= & {\left[A^{\top}(t) \hat{p}_{2}(t)+C^{\top}(t) \hat{q}_{2}(t)-Q_{2}(t) \hat{x}(t)\right] \mathrm{d} t-\hat{q}_{2}(t) W(t) } \\
\hat{x}(0)= & 0, \hat{p}_{2}(T)=-\Phi_{2} \hat{x}(T)
\end{aligned}\right.
$$


with

$$
\begin{aligned}
\hat{\varphi}_{2}\left(t, \hat{p}_{2}(t), \hat{q}_{2}(t)\right)= & \varphi_{2}\left(t, p_{2}^{1}(t), q_{2}^{1}(t)\right)-\varphi_{2}\left(t, p_{2}^{2}(t), q_{2}^{2}(t)\right) \\
= & \mathbf{P}_{\Gamma_{2}}\left[R_{2}^{-1}(t)\left(B_{2}^{\top}(t) p_{2}^{1}(t)+D_{2}^{\top}(t) q_{2}^{1}(t)\right)\right] \\
& -\mathbf{P}_{\Gamma_{2}}\left[R_{2}^{-1}(t)\left(B_{2}^{\top}(t) p_{2}^{2}(t)+D_{2}^{\top}(t) q_{2}^{2}(t)\right)\right]
\end{aligned}
$$

First, applying Itô's formula to $\langle\hat{p}, \hat{x}\rangle$ and taking expectations on both sides (noting the monotonicity property of $\widehat{\varphi}$, see Proposition A.4 , we have:

$$
\begin{aligned}
0= & \mathbb{E}\left\langle\Phi_{2} \hat{x}(T), \hat{x}(T)\right\rangle \\
& +\mathbb{E}\left[\int_{0}^{T}\left(\left\langle\left(B_{2}^{\top}(t) \hat{p}_{2}(t)+D_{2}^{\top}(t) \hat{q}_{2}(t)\right), \hat{\varphi}_{2}\left(t, \hat{p}_{2}(t), \hat{q}_{2}(t)\right)\right\rangle+\left\langle\hat{x}(t), Q_{2}(t) \hat{x}(t)\right\rangle\right) \mathrm{d} t\right] \\
\geq & \mathbb{E}\left\langle\Phi_{2} \hat{x}(T), \hat{x}(T)\right\rangle+\mathbb{E}\left[\int_{0}^{T}\left\langle\hat{x}(t), Q_{2}(t) \hat{x}(t)\right\rangle \mathrm{d} t\right]
\end{aligned}
$$

Thus, $\Phi_{2} \hat{x}(T)=0$ and $Q_{2}(t) \hat{x}(t)=0$ which implies $\hat{p}_{2}(t) \equiv 0, \hat{q}_{2}(t) \equiv 0$. Next, we have $\hat{\varphi}_{2}\left(t, \hat{p}_{2}(t), \hat{q}_{2}(t)\right) \equiv 0$ which further implies $\hat{x}(t) \equiv 0$. Hence the uniqueness follows.

(Existence) Consider a family of parameterized FBSDEs as follow: 4 :

$$
\left\{\begin{aligned}
\mathrm{d} x^{\alpha} & =\left[\alpha \mathbb{B}\left(x^{\alpha}, p_{2}^{\alpha}, q_{2}^{\alpha}\right)+\psi\right] \mathrm{d} t+\left[\alpha \mathbb{C}\left(x^{\alpha}, p_{2}^{\alpha}, q_{2}^{\alpha}\right)+\phi\right] \mathrm{d} W(t) \\
-\mathrm{d} p_{2}^{\alpha} & =\left[\alpha \mathbb{F}\left(x^{\alpha}, p_{2}^{\alpha}, q_{2}^{\alpha}\right)+\zeta\right] \mathrm{d} t-q_{2}^{\alpha} \mathrm{d} W(t) \\
x^{\alpha}(0) & =x_{0}, p_{2}^{\alpha}(T)=-\alpha \Phi_{2} \hat{x}(T)+\eta
\end{aligned}\right.
$$

with

$$
\left\{\begin{array}{l}
\mathbb{B}\left(t, x, p_{2}, q_{2}\right)=A x+B_{2} \varphi_{2}\left(t, p_{2}, q_{2}\right) \\
\mathbb{C}\left(t, x, p_{2}, q_{2}\right)=C x+D_{2}(t) \varphi_{2}\left(t, p_{2}, q_{2}\right) \\
\mathbb{F}\left(t, x, p_{2}, q_{2}\right)=A^{\top} p_{2}+C^{\top} q_{2}-Q_{2} x
\end{array}\right.
$$

Here, $(\psi, \phi, \zeta)$ are given processes in $\mathcal{M}^{2}\left(0, T ; \mathbb{R}^{n}\right) \times \mathcal{M}^{2}\left(0, T ; \mathbb{R}^{n}\right) \times \mathcal{M}^{2}\left(0, T ; \mathbb{R}^{n}\right)$, and $\eta$ is a $\mathbb{R}^{n}$-valued square integrable random variable which is $\mathcal{F}_{T}$-measurable. When $\alpha=0$, we have a decoupled FBSDEs whose solvability is trivial:

$$
\left\{\begin{aligned}
\mathrm{d} x & =\psi \mathrm{d} t+\phi \mathrm{d} W(t) \\
-\mathrm{d} p_{2} & =\zeta \mathrm{d} t-q_{2} \mathrm{~d} W(t) \\
x(0) & =x_{0}, p_{2}(T)=\eta
\end{aligned}\right.
$$

Denote

$$
\widetilde{\mathcal{M}}(0, T)=\mathcal{M}^{2}\left(0, T ; \mathbb{R}^{n}\right) \times \mathcal{M}^{2}\left(0, T ; \mathbb{R}^{n}\right) \times \mathcal{M}^{2}\left(0, T ; \mathbb{R}^{n}\right) .
$$

Now we introduce a mapping $I_{\alpha_{0}}:\left(x, p_{2}, q_{2}\right) \in \widetilde{\mathcal{M}}(0, T) \longrightarrow\left(X, P_{2}, Q_{2}\right) \in \widetilde{\mathcal{M}}(0, T)$ via the following FBSDEs:

$$
\left\{\begin{aligned}
\mathrm{d} X= & {\left[\alpha_{0} \mathbb{B}\left(X, P_{2}, Q_{2}\right)+\delta \mathbb{B}\left(x, p_{2}, q_{2}\right)+\psi\right] \mathrm{d} t } \\
& +\left[\alpha_{0} \mathbb{C}\left(X, P_{2}, Q_{2}\right)+\delta \mathbb{C}\left(x, p_{2}, q_{2}\right)+\phi\right] \mathrm{d} W(t) \\
-\mathrm{d} P_{2}= & {\left[\alpha_{0} \mathbb{F}\left(X, P_{2}, Q_{2}\right)+\delta \mathbb{F}\left(x, p_{2}, q_{2}\right)+\zeta\right] \mathrm{d} t-Q \mathrm{~d} W(t) } \\
X(0)= & x_{0}, P(T)=-\alpha_{0} \Phi_{2} X(T)-\delta \Phi_{2} x(T)+\eta
\end{aligned}\right.
$$

Considering $I_{\alpha_{0}}:\left(x, p_{2}, q_{2}\right) \longrightarrow\left(X, P_{2}, Q_{2}\right)$ and $I_{\alpha_{0}}:\left(x^{\prime}, p_{2}^{\prime}, q_{2}^{\prime}\right) \longrightarrow\left(X^{\prime}, P_{2}^{\prime}, Q_{2}^{\prime}\right)$ and

$$
\begin{aligned}
(\widehat{x}, \widehat{p}, \widehat{q}) & =\left(x-x^{\prime}, p_{2}-p_{2}^{\prime}, q_{2}-q_{2}^{\prime}\right), \\
(\widehat{X}, \widehat{P}, \widehat{Q}) & =\left(X-X^{\prime}, P_{2}-P_{2}^{\prime}, Q_{2}-Q_{2}^{\prime}\right)
\end{aligned}
$$

\footnotetext{
${ }^{4}$ For simplicity, the dependence of coefficients on time variable $t$ is suppressed.
} 
with

$$
\left\{\begin{aligned}
\mathrm{d} \widehat{X}= & {\left[\alpha_{0} \widehat{\mathbb{B}}(\widehat{X}, \widehat{P}, \widehat{Q})+\delta \widehat{\mathbb{B}}(\widehat{x}, \widehat{p}, \widehat{q})\right] \mathrm{d} t } \\
& +\left[\alpha_{0} \widehat{\mathbb{C}}(\widehat{X}, \widehat{P}, \widehat{Q})+\delta \widehat{\mathbb{C}}(\widehat{x}, \widehat{p}, \widehat{q})\right] \mathrm{d} W(t) \\
-\mathrm{d} \widehat{P}= & {\left[\alpha_{0} \widehat{\mathbb{F}}(\widehat{X}, \widehat{P}, \widehat{Q})+\delta \widehat{\mathbb{F}}(\widehat{x}, \widehat{p}, \widehat{q})\right] \mathrm{d} t-\widehat{Q} \mathrm{~d} W(t) } \\
\widehat{X}(0)= & 0, \widehat{P}(T)=-\alpha_{0} \Phi_{2} \widehat{X}(T)-\delta \Phi_{2} \widehat{x}(T)
\end{aligned}\right.
$$

$$
\left\{\begin{array}{l}
\widehat{\mathbb{B}}(\widehat{X}, \widehat{P}, \widehat{Q})=\mathbb{B}\left(X, P_{2}, Q_{2}\right)-\mathbb{B}\left(X, P_{2}^{\prime}, Q_{2}^{\prime}\right), \\
\widehat{\mathbb{C}}(\widehat{X}, \widehat{P}, \widehat{Q})=\mathbb{C}\left(X, P_{2}, Q_{2}\right)-\mathbb{C}\left(X, P_{2}^{\prime}, Q_{2}^{\prime}\right), \\
\widehat{\mathbb{F}}(\widehat{X}, \widehat{P}, \widehat{Q})=\mathbb{F}\left(X, P_{2}, Q_{2}\right)-\mathbb{F}\left(X, P_{2}^{\prime}, Q_{2}^{\prime}\right),
\end{array}\right.
$$

Applying Itô formula to $\langle\widehat{P}, \widehat{X}\rangle$ and taking expectations on both sides:

$$
\begin{aligned}
& \mathbb{E}\left\langle\widehat{X}(T),-\alpha_{0} \Phi_{2} \widehat{X}(T)-\delta \Phi_{2} \widehat{x}(T)\right\rangle \\
= & \mathbb{E}\left\{\int _ { 0 } ^ { T } \left[\left\langle\widehat{X}(s),-\alpha_{0} \widehat{\mathbb{F}}(\widehat{X}(s), \widehat{P}(s), \widehat{Q}(s))\right\rangle+\langle\widehat{X}(s),-\delta \widehat{\mathbb{F}}(\widehat{x}(s), \widehat{p}(s), \widehat{q}(s))\rangle\right.\right. \\
+ & \left\langle\widehat{P}(s), \alpha_{0} \widehat{\mathbb{B}}(\widehat{X}(s), \widehat{P}(s), \widehat{Q}(s))\right\rangle+\langle\widehat{P}(s), \delta \widehat{\mathbb{B}}(\widehat{x}(s), \widehat{p}(s), \widehat{q}(s))\rangle \\
& \left.\left.+\left\langle\widehat{Q}(s), \alpha_{0} \widehat{\mathbb{C}}(\widehat{X}(s), \widehat{P}(s), \widehat{Q}(s))\right\rangle+\langle\widehat{Q}(s), \delta \widehat{\mathbb{C}}(\widehat{x}(s), \widehat{p}(s), \widehat{q}(s))\rangle\right] \mathrm{d} s\right\}
\end{aligned}
$$

Rearranging the above terms, we have

$$
\begin{aligned}
& \alpha_{0} \mathbb{E}\left\langle\widehat{X}(T), \Phi_{2} \widehat{X}(T)\right\rangle+\alpha_{0} \mathbb{E}\left\{\int_{0}^{T}[\langle\widehat{X}(s),-\widehat{\mathbb{F}}(\widehat{X}(s), \widehat{P}(s), \widehat{Q}(s))\rangle\right. \\
& +\langle\widehat{P}(s), \widehat{\mathbb{B}}(\widehat{X}(s), \widehat{P}(s), \widehat{Q}(s))\rangle+\langle\widehat{Q}(s), \widehat{\mathbb{C}}(\widehat{X}(s), \widehat{P}(s), \widehat{Q}(s))\rangle] \mathrm{d} s\} \\
= & \delta \mathbb{E}\left\{\int_{0}^{T}[\langle\widehat{X}(s), \widehat{\mathbb{F}}(\widehat{x}(s), \widehat{p}(s), \widehat{q}(s))\rangle-\langle\widehat{P}(s), \widehat{\mathbb{B}}(\widehat{x}(s), \widehat{p}(s), \widehat{q}(s))\rangle\right. \\
& -\langle\widehat{Q}(s), \delta \widehat{\mathbb{C}}(\widehat{x}(s), \widehat{p}(s), \widehat{q}(s))\rangle] \mathrm{d} s\}-\delta \mathbb{E}\left\langle\widehat{X}(T), \Phi_{2} \widehat{x}(T)\right\rangle
\end{aligned}
$$

Hence,

$$
\begin{aligned}
& \alpha_{0} \mathbb{E}\left|\Phi_{2}^{\frac{1}{2}} \widehat{X}(T)\right|^{2}+\alpha_{0} \mathbb{E}\left\{\int_{0}^{T}\left|Q_{2}^{\frac{1}{2}}(s) \widehat{X}(s)\right|^{2} \mathrm{~d} s\right\} \\
\leq & \alpha_{0} \mathbb{E}\left\langle\widehat{X}(T), \Phi_{2} \widehat{X}(T)\right\rangle+\alpha_{0} \mathbb{E}\left\{\int_{0}^{T}[\langle\widehat{X}(s),-\widehat{\mathbb{F}}(\widehat{X}(s), \widehat{P}(s), \widehat{Q}(s))\rangle\right. \\
& +\langle\widehat{P}(s), \widehat{\mathbb{B}}(\widehat{X}(s), \widehat{P}(s), \widehat{Q}(s))\rangle+\langle\widehat{Q}(s), \widehat{\mathbb{C}}(\widehat{X}(s), \widehat{P}(s), \widehat{Q}(s))\rangle] \mathrm{d} s\} \\
= & \delta \mathbb{E}\left\{\int_{0}^{T}[\langle\widehat{X}(s), \widehat{\mathbb{F}}(\widehat{x}(s), \widehat{p}(s), \widehat{q}(s))\rangle-\langle\widehat{P}(s), \widehat{\mathbb{B}}(\widehat{x}(s), \widehat{p}(s), \widehat{q}(s))\rangle\right. \\
& -\langle\widehat{Q}(s), \delta \widehat{\mathbb{C}}(\widehat{x}(s), \widehat{p}(s), \widehat{q}(s))\rangle] \mathrm{d} s\}-\delta \mathbb{E}\left\langle\widehat{X}(T), \Phi_{2} \widehat{x}(T)\right\rangle \\
\leq & \delta \mathbb{E}\left\{\int_{0}^{T}\left[|\widehat{X}(s)|^{2}+|\widehat{P}(s)|^{2}+|\widehat{Q}(s)|^{2}\right] \mathrm{d} s\right\} \\
& +\delta \mathbb{E}\left\{\int_{0}^{T}\left[|\widehat{x}(s)|^{2}+|\widehat{p}(s)|^{2}+|\widehat{q}(s)|^{2}\right] \mathrm{d} s\right\} \\
& +\delta C\left(|\widehat{x}(T)|^{2}+|\widehat{X}(T)|^{2}\right) .
\end{aligned}
$$


We point out that, the first inequality uses the monotonicity property of $\varphi(p, q)$ (Proposition A.2). The second inequality is due to the basic geometric inequality and Lipschitz property of projection operator (Proposition A.3).

Then, by standard estimates of BSDE:

$$
\begin{aligned}
& \mathbb{E}\left\{\int_{0}^{T}\left[|\widehat{P}(s)|^{2}+|\widehat{Q}(s)|^{2}\right] \mathrm{d} s\right\} \\
\leq & \delta C \mathbb{E}\left\{\int_{0}^{T}\left[|\widehat{x}(s)|^{2}+|\widehat{p}(s)|^{2}+|\widehat{q}(s)|^{2}\right] \mathrm{d} s\right\}+\delta C \mathbb{E}|\widehat{x}(T)|^{2} \\
& +C \alpha_{0}\left(\mathbb{E}\left|\Phi_{2}^{\frac{1}{2}} \widehat{X}(T)\right|^{2}+\mathbb{E}\left\{\int_{0}^{T}\left|Q_{2}^{\frac{1}{2}}(s) \widehat{X}(s)\right|^{2} \mathrm{~d} s\right\}\right) .
\end{aligned}
$$

Next, by the standard estimate of forward SDEs:

$$
\begin{aligned}
& \mathbb{E}\left\{\int_{0}^{T}|\widehat{X}(s)|^{2} \mathrm{~d} s\right\}+\mathbb{E}|\widehat{X}(T)|^{2} \\
\leq & \delta C \mathbb{E}\left\{\int_{0}^{T}\left[|\widehat{x}(s)|^{2}+|\widehat{p}(s)|^{2}+|\widehat{q}(s)|^{2}\right] \mathrm{d} s\right\} \\
& +C \mathbb{E}\left[\int_{0}^{T}\left[|\widehat{P}(s)|^{2}+|\widehat{Q}(s)|^{2}\right] \mathrm{d} s\right]+\delta C \mathbb{E}|\widehat{x}(T)|^{2} .
\end{aligned}
$$

Based on the above estimates (59)-(61), we see the mapping $I$ satisfying

$$
\mathbb{E} \int_{0}^{T}\left(\left|\widehat{X}_{s}\right|^{2}+\left|\widehat{P}_{s}\right|^{2}+\left|\widehat{Q}_{s}\right|^{2}\right) d s+\mathbb{E}\left|\widehat{X}_{T}\right|^{2} \leq K \delta\left(\mathbb{E} \int_{0}^{T}\left(\left|\widehat{x}_{s}\right|^{2}+\left|\widehat{p}_{s}\right|^{2}+\left|\widehat{q}_{s}\right|^{2}\right) d s+\mathbb{E}\left|\widehat{x}_{T}\right|^{2}\right) .
$$

It follows the mapping is a contraction and the existence follows immediately using the arguments presented in [28] and 29].

\section{Discussion on Riccati equation}

Recall the stochastic Hamilton system (taken from Tang [22]) is given by

$$
\left\{\begin{aligned}
\mathrm{d} x(t) & =(A(t) x(t)+B(t) u(t)) \mathrm{d} t+\sum_{i=1}^{d}\left(C^{i}(t) x(t)+D^{i}(t) u(t)\right) \mathrm{d} W^{i}(t), \\
u(t) & =-N^{-1}(t)\left[B^{\top}(t) y(t)+\sum_{i=1}^{d} D^{i}(t)^{\top} z^{i}(t)\right], \\
-\mathrm{d} y(t) & =\left[A^{\top}(t) y(t)+\sum_{i=1}^{d} C^{i}(t)^{\top} z^{i}(t)+Q(t) x(t)\right] \mathrm{d} t-\sum_{i=1}^{d} z^{i}(t) \mathrm{d} W^{i}(t) \\
x(\tau) & =h \in L^{2}\left(\Omega, \mathcal{F}_{\tau}, P ; \mathbb{R}^{n}\right), y(T)=M x(T) .
\end{aligned}\right.
$$

Inserting $u(\cdot)$ into the first equation, we have

$$
\left\{\begin{aligned}
\mathrm{d} x= & \left(A x-B N^{-1}\left[B^{\top} y+\sum_{i=1}^{d}\left(D^{i}\right)^{\top} z^{i}\right]\right) \mathrm{d} t \\
& +\sum_{i=1}^{d}\left(C^{i} x-D^{i} N^{-1}\left[B^{\top} y+\sum_{i=1}^{d}\left(D^{i}\right)^{\top} z^{i}\right]\right) \mathrm{d} W^{i}(t) \\
-\mathrm{d} y= & {\left[A^{\top} y+\sum_{i=1}^{d}\left(C^{i}\right)^{\top} z^{i}+Q x\right] \mathrm{d} t-\sum_{i=1}^{d} z^{i} \mathrm{~d} W^{i}(t) } \\
x(\tau)= & h \in L^{2}\left(\Omega, \mathcal{F}_{\tau}, P ; \mathbb{R}^{n}\right), y(T)=M x(T)
\end{aligned}\right.
$$

A formal approach to derive the associated Riccati equation from the stochastic Hamilton system a priori assumes that there is a semi-martingale $K$ of the form

$$
K(t)=K(0)-\int_{0}^{t} K_{1}(s) \mathrm{d} s+\int_{0}^{t} \sum_{i=1}^{d} L^{i}(s) \mathrm{d} W(s), 0 \leq t \leq T .
$$


such that

$$
y(t)=K(t) x(t)
$$

Then, applying Itô's formula to $K(t) x(t)$, we have

$$
\begin{aligned}
& K\left(A x-B N^{-1}\left[B^{\top} y+\sum_{i=1}^{d}\left(D^{i}\right)^{\top} z^{i}\right]\right) \mathrm{d} t \\
& +K \sum_{i=1}^{d}\left(C^{i} x-D^{i} N^{-1}\left[B^{\top} y+\sum_{i=1}^{d}\left(D^{i}\right)^{\top} z^{i}\right]\right) \mathrm{d} W^{i}(t) \\
& +K_{1} x \mathrm{~d} t+\sum_{i=1}^{d} L^{i} x \mathrm{~d} W^{i}(t) \\
& +\sum_{i=1}^{d} L^{i}(s) \sum_{i=1}^{d}\left(C^{i} x-D^{i} N^{-1}\left[B^{\top} y+\sum_{i=1}^{d}\left(D^{i}\right)^{\top} z^{i}\right]\right) \mathrm{d} t \\
& =\quad \mathrm{d} y(t) \\
& =-\left[A^{\top} y+\sum_{i=1}^{d}\left(C^{i}\right)^{\top} z^{i}+Q x\right] \mathrm{d} t+\sum_{i=1}^{d} z^{i} \mathrm{~d} W^{i}(t) .
\end{aligned}
$$

It follows that

$$
z^{i}=K\left(C^{i} x-D^{i} N^{-1} B^{\top} K x-D^{i} N^{-1}\left(D^{i}\right)^{\top} z^{i}\right)+L^{i} x, 1 \leq i \leq d .
$$

Immediately,

$$
z^{i}=Z^{i} x
$$

where

$$
Z^{i}=\left(I+K D^{i} N^{-1}\left(D^{i}\right)^{\top}\right)^{-1}\left(K C^{i}-K D^{i} N^{-1} B^{\top} K+L^{i}\right) .
$$

Substituting (63) 5 into (62), we identify the integrands of the Lebesgue integral. As a consequence

$$
\begin{aligned}
& K A-K \underbrace{B N^{-1} B^{\top}}_{\boldsymbol{B}_{1}} K-K \underbrace{B N^{-1} \sum_{i=1}^{d}\left(D^{i}\right)^{\top} Z^{i}-K_{1}}_{\boldsymbol{B}_{2}} \\
& +\sum_{i=1}^{d} L^{i}(s) \sum_{i=1}^{d}(C^{i}-\underbrace{D^{i} N^{-1} B^{\top}}_{\boldsymbol{D}_{1}} K-\underbrace{D^{i} N^{-1}\left(D^{i}\right)^{\top}}_{\boldsymbol{D}_{2}} Z^{i}) \\
& =-A^{\top} K-\sum_{i=1}^{d}\left(C^{i}\right)^{\top} Z^{i}-Q .
\end{aligned}
$$

\footnotetext{
${ }^{5}$ In order to get the standard form $(3.1)$ in Tang [22, the rest proceeding will employ the well-known matrix inverse formula, that is,

$$
(A+B C D)^{-1}=A^{-1}-A^{-1} B\left(D A^{-1} B+C^{-1}\right)^{-1} D A^{-1}
$$

where $A^{-1}, C^{-1}$, and either $(A+B C D)^{-1}$ or $\left(D A^{-1} B+C^{-1}\right)^{-1}$ are assumed to exist.
} 
Now

$$
\begin{aligned}
K_{1}= & A^{\top} K+\sum_{i=1}^{d}\left(C^{i}\right)^{\top} Z^{i}+Q+K A-K \boldsymbol{B}_{1} K-K \boldsymbol{B}_{2} Z^{i} \\
& +\sum_{i=1}^{d} L^{i}(s) \sum_{i=1}^{d}\left(C^{i}-\boldsymbol{D}_{1} K-\boldsymbol{D}_{2} Z^{i}\right) .
\end{aligned}
$$

This is another expression for Riccati equation (3.1) in Tang [22].

\section{References}

[1] H. von Stackelberg, Marktform und Gleichgewicht, Springer-Verlag, Wien New York, 1934 (in German); Market Structure and Equilibrium, Springer-Verlag, Berlin Heidelberg, 2011 (in English).

[2] B. Øksendal, L. Sandal, and J. Uboe, Stochastic Stackelberg equilibria with applications to time dependent newsvendor models. J. Econ. Dyna. \& Control, 37(7), 1284-1299, (2013).

[3] L. Chen, Y. Shen, On a new paradigm of optimal reinsurance: A stochastic Stackelberg differential game between an insurer and a reinsurer. ASTIN Bulletin, 48(2), 905-960, 2018.

[4] T. Li, S. P. Sethi, A review of dynamic Stackelberg game models. Discrete Contin. Dyn. Syst., Ser. B, 22(1), 125-159, 2017.

[5] J. Cvitanić, J. F. Zhang, Contract Theory in Continuous-Time Models, Springer-Verlag, Berlin, 2013.

[6] A. Chutani, Sethi. S. P. Optimal advertising and pricing in a dynamic durable goods supply chain. Journal of Optimization Theory and Applications 154 615-643 (2012).

[7] A. Chutani, Sethi. S. P. Cooperative advertising in a dynamic retail market oligopoly. Dynamic Games and Applications 2 347-375 (2012).

[8] E. Dockner, JØgensen, S. N. V. Long, G. Sorger. Differential Games in Economics and Management Science. Cambridge University Press, Cambridge, UK. (2000).

[9] X. He, A. Prasad, S. P. Sethi. Cooperative advertising and pricing in a dynamic stochastic supply chain: feedback stackelberg strategies. Production and Operations Management 18 78-94. (2009).

[10] X. He, A. Prasad, S. P. Sethi, G. J. Gutierrez. A survey of Stackelberg differential game models in supply and marketing channels. Journal of Systems Science and Systems Engineering 16 385-413 (2007).

[11] K. Kogan, C. S. Tapiero. Supply Chain Games: Operations Management and Risk Valuation. Springer, New York (2007).

[12] G. Wang, Z. Yu. A Pontryagin's maximum principle for non-zero sum differential games of backward stochastic differential equations with applications. IEEE Trans. Auto. Contr., 55(7), 1742-1747, 2010.

[13] G. Wang, Z. Yu, A partial information non-zero sum differential game of backward stochastic differential equations with applications. Automatica, 2012, 48(2),342-352. 
[14] Z. Yu, An optimal feedback control-strategy pair for zero-sum linear-quadratic stochastic differential game: the Riccati equation approach, SIAM Journal on Control and Optimization, 53 (2015), no. 4, 2141-2167.

[15] D. Castanon, M. Athans, On stochastic dynamic Stackelberg strategies. Automatica, 12(2), 177-183, 1976.

[16] J. Yong. A leader-follower stochastic linear quadratic differential game. SIAM Journal on Control and Optimization 41 1015-1041, (2002).

[17] T. Başar, A. Bensoussan, and S. P. Sethi, Differential games with mixed leadership: The open-loop solution. Appl. Math. Comput., 217(3), 972-979, (2010).

[18] A. Bensoussan, S. Chen and S.P. Sethi. The Maximum Principle for Global Solutions of Stochastic Stackelberg Differential Games, SIAM J. Control Optim., 53(4), 1956-1981 (2015).

[19] H. Mukaidani, H. Xu, Stackelberg strategies for stochastic systems with multiple followers. Automatica, 53, 53-79, (2015).

[20] N. Li, Z. Y. Yu, Forward-backward stochastic differential equations and linear-quadratic generalized Stackelberg games. SIAM J. Control Optim., 56(6), 4148-4180, (2018).

[21] J. H. Huang, K. H. Si, and Z. Wu, Linear-quadratic mixed Stackelberg-Nash stochastic differential game with major-minor agents. Appl. Math. Opt., https://doi.org/10.1007/s00245020-09713-z.

[22] S. Tang, General linear quadratic optimal stochastic control problems with random coefficients: linear stochastic hamilton systems and backward stochastic Riccati equations, SIAM J. Control Optim. 42(1):53-75, (2003).

[23] Y. Hu and X.Y. Zhou. Constrained stochastic LQ control with random coefficients, and application to portfolio selection. SIAM Journal on Control and Optimization, 44, 444-466 (2005).

[24] Z. Wu. Maximum principle for optimal control problem of fully coupled forward-backward stochastic systems. Systems Science and Mathematical Science, Vol. 11 No.3 Jul. (1998).

[25] J. Yong, X. Y. Zhou, Stochastic Controls. Hamiltonian Systems and HJB Equations, Springer-Verlag, New York, (1999).

[26] S. Peng, A general stochastic maximum principle for optimal control problems, SIAM J. Control $\& 3$ Optim., 28, 966-979, (1990).

[27] F. Antonelli, Backward-forward stochastic differential equations, Ann. Appl. Probab., 3 (1993),777-793.

[28] Y. Hu and S. Peng. Solutions of forward-backward stochastic differential equations. Probability Theory and Related Fields, 103, 273-283 (1995).

[29] S. Peng, Z. Wu. Fully Coupled Forward-backward Stochastic Differential Equations and Applications to Optimal Control. SIAM Journal on Control and Optimization, 37 825-843 (1999).

[30] D. Duffie and L. Epstein, Asset pricing with stochastic differential utilities, Rev. Financial Stud, 5 (1992), 411-436. 
[31] Z. Wu and Z. Yu, Probabilistic interpretation for a system of quasilinear parabolic partial differential equation combined with algebra equations, Stochastic Processes and their Applications, 124 (2014), no. 12, 3921-3947.

[32] J. T. Shi, G. C. Wang, and J. Xiong, Leader-follower stochastic differential game with asymmetric information and applications. Automatica, 63, 60-73, 2016.

[33] J. T. Shi, G. C. Wang, and J. Xiong, Linear-quadratic stochastic Stackelberg differential game with asymmetric information. Sci. China Infor. Sci., 60, 092202:1-15, 2017.

[34] H. Brezis. Functional Analysis, Sobolev Spaces and Partial Differential Equations. Springer, New York, (2011). 